\section{D) Check for updates}

Cite this: Inorg. Chem. Front., 2020, 7, 1801
Received 11th February 2020, Accepted 26th March 2020

DOI: 10.1039/d0qi00167h

rsc.li/frontiers-inorganic

\title{
Luminescent metal-organic frameworks (LMOFs) as potential probes for the recognition of cationic water pollutants
}

\author{
Partha Samanta, (D) a Sumanta Let, (D) $\dagger^{a}$ Writakshi Mandal, (D) $\dagger^{a}$ Subhajit Dutta (D) ${ }^{a}$ \\ and Sujit K. Ghosh (iD *a,b
}

\begin{abstract}
Metal-organic frameworks (MOFs) are a unique class of crystalline extended networks with a tuneable architecture. Accordingly, due to their tuneable pores and high surface area, MOFs have gained increasing interest in the last two decades. Luminescent MOF (LMOF)-based sensory materials have provided an excellent platform for the detection of a wide range of toxic, hazardous and biologically important species. Furthermore, the increasing environmental pollution has become an imminent threat to life on Earth. However, the detection of pollutants even at very low levels is still a challenge in the scientific community. Thus, several MOF-based probes have been developed for the sensing of toxic and hazardous species in recent years. Herein, we summarize the MOF-based sensing of cationic organic and inorganic water pollutants based on the recent reports.
\end{abstract}

\section{Introduction}

Global urbanization and growing industries are an integral part of development worldwide. However, such worldwide

${ }^{a}$ Department of Chemistry, Indian Institute of Science Education and Research, Dr Homi Bhabha Road, Pashan, Pune 411008, India. E-mail: sghosh@iiserpune.ac.in; Tel: +912025908076

${ }^{b}$ Centre for Energy Science, IISER PUne, Dr Homi Bhabha Road, Pashan, Pune 411008, India

$\dagger$ These authors contributed equally. urbanization and development have led to the adverse effect of increasing environmental pollution. ${ }^{1}$ In addition, the global environment has a direct impact on human life and other living organisms. Consequently, the gradual increase in the environmental pollution has affected the life of humans and has become a huge threat to every living system. Among the various types of environmental pollution, water pollution has been considered as one of the life-threatening and lethal issues in recent years. ${ }^{2}$ According to the World Health Organisation (WHO), contaminated drinking water has been found to cause almost 485000 diarrhoeal deaths each year. ${ }^{3}$

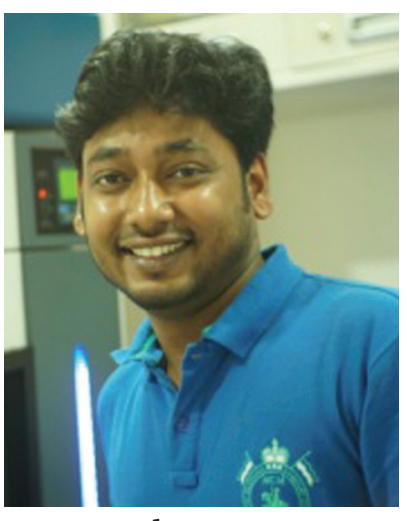

Partha Samanta
Partha Samanta obtained his B.Sc. from the University of Calcutta in 2011. After that, he pursued his M.Sc. at the Indian Institute of Technology (IIT), Kharagpur. After obtaining his M.Sc., he moved to the Indian Institute of Science Education and Research (IISER), Pune to pursue his doctoral studies, where he joined the research group of Dr Sujit K. Ghosh in 2013. Since then he has been exploring various porous organic materials toward energy and a wide variety of environmental applications.

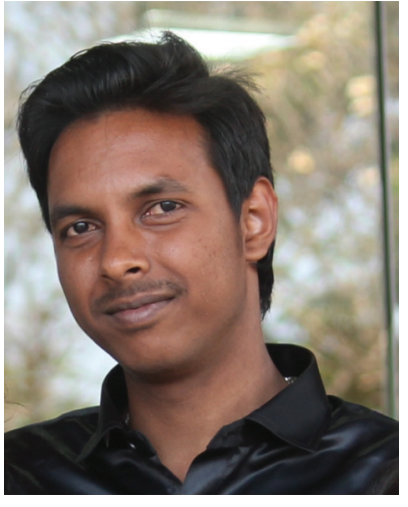

Sumanta Let
Sumanta Let received his B.Sc. from the University of Calcutta (2014), after which he enrolled in the Indian Institute of Technology (ISM), Dhanbad to obtain an M.Sc. Upon completion of his M.Sc. (2016), he enrolled in the Doctoral Program at IISER, Pune under the supervision of Dr Sujit K. Ghosh. Sumanta's research focuses on the design and development of function-based porous materials for the remediation of pollutants in air and water. 
Furthermore, it has been observed that a large number of the population (at least 2 billion) regularly use drinking water from contaminated sources. In addition, this scenario is expected to be even worse in coming years. ${ }^{4}$ Due to the pollution of surface water and groundwater, the amount of fresh drinking water has become limited. It has been estimated that half of the total population of world will be living in water stressed areas by 2025. ${ }^{3}$ There are different types of sources of water pollution, where among them, chemical sources are considered as one of the most toxic and hazardous way of water contamination. Chemical sources can be classified into two major categories, inorganic pollutants and organic pollutants. On the other hand, depending on their electronic charge, chemical pollutants can be categorized into neutral, anionic and cationic pollutants. Inorganic metal cations (such as heavy metal cations and transition metal cations) and several organic cations have become a concern due to the heavy water pollution caused by them and their ill-effects. Especially, water pollution due to heavy metal ions $\left(\mathrm{Hg}^{2+}, \mathrm{Pb}^{2+}, \mathrm{Cd}^{2+}\right.$, etc. $)$ has attracted tremendous attention in recent years because of their very high toxicity and acute poisoning. ${ }^{5}$ It has been observed that the aforementioned heavy metal ions cause severe health hazards even when they are present at the parts per billion (ppb) level in water bodies. ${ }^{6}$ The most toxic heavy metal, $\mathrm{Hg}$ (II) ion, can damage several body parts, including the brain, kidney, spinal cord, liver, etc. together with cognitive and motion disorders. ${ }^{7,8}$ Furthermore, $\mathrm{Hg}$ (II) ions can lead to the lethal Minamata disease. Likewise, lead $(\mathrm{Pb})$ poisoning has been found to cause several diseases, including anemia and epileptic seizures, and can also lead to a coma or often death. ${ }^{9}$ Besides heavy metal ions, some transition metal ions (such as $\mathrm{Fe}^{3+}$, and $\mathrm{Cu}^{2+}$ ) in excess of the permissible limit have also been considered as toxic water pollutants. On the other hand, several organic cationic compounds (such as paraquat and diquat) have been identified as highly toxic and hazardous water contaminants. ${ }^{10}$ Often these organic compounds are used as herbicides in different farms, which eventually pollute various water effluents or sewage. This type of organic con- taminant has been observed to be highly fatal and can lead to damage of the kidney, eye, lung, heart, liver, etc., and even death. It has been estimated that paraquat toxicity causes almost 5000 deaths in China alone each year. ${ }^{11}$ Thus, understanding the conditions and consequences of this type of water pollution and monitoring of these water contaminants become an urgent issue worldwide.

Consequently, the detection of metal cations has been considered essential, not only for tackling environmental issues but also in monitoring the concentration of different metal cations in the human body. Metal ions such as $\mathrm{Fe}^{3+}, \mathrm{Fe}^{2+}$, $\mathrm{Cu}^{2+}, \mathrm{Na}^{+}$, and $\mathrm{K}^{+}$are also useful elements for humans, but they should only be present in the human body at a certain concentration. ${ }^{12,13}$ Accordingly, it has been observed that transition metals (such as $\mathrm{Fe}^{3+}, \mathrm{Fe}^{2+}$, and $\mathrm{Cu}^{2+}$ ) can also cause several issues in the human body when they are present in excess of their respective permissible limits. Moreover, deviation from the respective particular concentration ranges of metal ions can result in serious diseases. Consequently, the detection of metal cations with high accuracy and at very low concentration levels is very important.

To date, several techniques have been employed for the detection of cationic pollutants, which include photoluminescence spectroscopy, inductively coupled plasma mass spectrometry (ICPMS), chromatography, X-ray absorption spectroscopy, colorimetric detection, and anodic stripping voltammetry. ${ }^{14}$ Among them, photoluminescence (PL) spectroscopy has been found to be an excellent platform for the detection of cationic water pollutants (Fig. 1). This is due to its advantageous features such as high sensitivity, quick response, ability to detect a trace amount of analyte, simple operation, and cost-effectiveness. Additionally, by employing photoluminescence spectroscopy, different types of materials have been reported in the literature for the detection of inorganic and organic cationic species. ${ }^{15,16}$ Recent years have witnessed the emergence of porous materials as suitable candidates as sensory probes. ${ }^{17}$ In the family of porous materials, metalorganic frameworks (MOFs) have gained paramount interest in

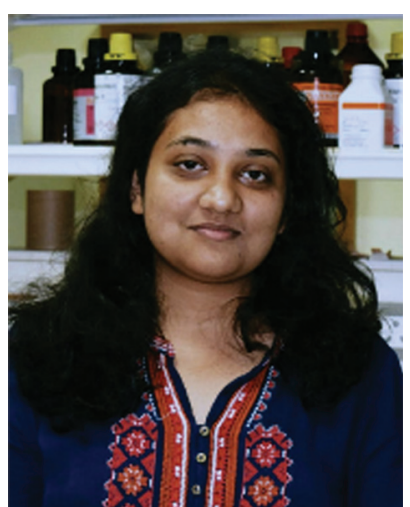

Writakshi Mandal
Writakshi Mandal obtained her B.Sc. (Chemistry) from the University of Calcutta in 2014 and received her M.Sc. Degree from Banaras Hindu University in 2016. She joined the Indian Institute of Science Education and Research (IISER), Pune in 2017 as a PhD student under the supervision of Dr Sujit K. Ghosh. Her research area focuses on the synthesis and functional studies of porous organic materials for sensing and sorption-based applications.

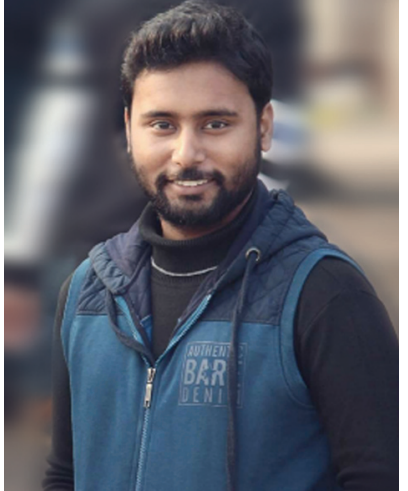

Subhajit Dutta
Subhajit Dutta obtained his B.Sc. (Chemistry) from the University of Calcutta in 2014 and received his M.Sc. Degree from the University of Calcutta in 2016. He joined the Indian Institute of Science Education and Research (IISER), Pune in 2016 as a PhD student under the supervision of Dr Sujit K. Ghosh. His research is centered on the synthesis and functional studies of porous materials for capture and sensing-based applications. 


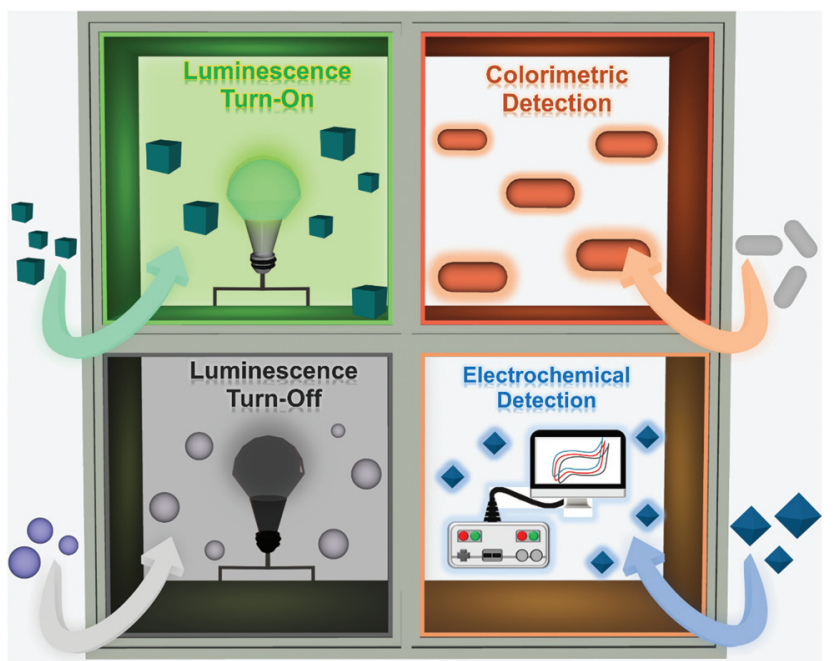

\section{MOF Based Different Sensing Methods}

Fig. 1 Schematic representation of the various metal-organic framework (MOF)-based sensing methods.

last two decades. Since they are constructed using organic linkers and metal nodes through non-covalent interactions, crystalline MOFs can serve as a suitable platform for a wide range of applications. ${ }^{18-22}$ Owing to their high surface area, tuneable porosity, tuneable pore surface, etc., MOFs have attracted significant attention from researchers. ${ }^{23-25}$ Additionally, the crystalline nature of MOFs provides suitable structure property correlation, which has proven to be essential for the modification of materials according to the targeted application. MOFs have been explored in the fields of gas capture, gas and solvent separation, drug delivery, fuel cell applications, sensing, electrochemical applications, etc. ${ }^{26-44}$ On account of their porosity and tuneable pore surface, MOFs

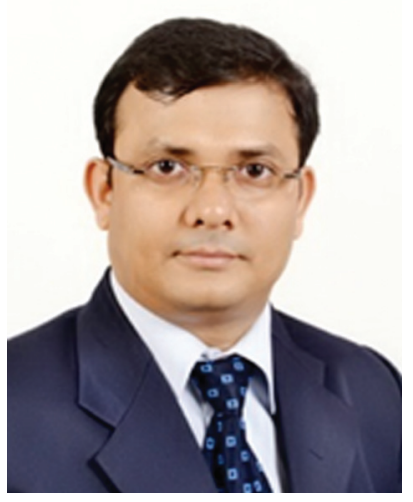

Sujit K. Ghosh
Dr Sujit K. Ghosh completed his PhD from the Indian Institute of Technology (IIT), Kanpur in 2006 under the supervision of Prof. Parimal K. Bharadwaj, after which he went to Kyoto University, Japan as a JSPS and CREST Postdoctoral Fellow (Host: Prof. Susumu Kitagawa). He is presently an Associate Professor in the Department of Chemistry at IISER, Pune. His current research work is primarily focused on advanced functional porous materials (such as metal-organic frameworks, metal-organic polyhedra, and porous organic polymers) aimed at industrial and environmental applications, especially sensing and sequestration of toxic and hazardous pollutants. have been considered strong candidates as sensory materials. The porosity of MOFs provides a pre-concentration effect, which can lead to the enhancement of the sensitivity of probes toward analytes. ${ }^{45}$ Thus, MOFs have been employed as sensory probes for various analytes, which include cationic, anionic and neutral species. ${ }^{45-56}$ Additionally, often bulk MOFs can be downsized to the nanometer $s$ to attain nanoscale MOFs that offer advantages over bulk MOF crystals. Furthermore, nanoscale MOFs exhibit versatility in composition, structure and have greater dispersity in water, which make them attractive materials for sensing applications. ${ }^{57-59}$ Alternatively, LMOFs have also been used to accommodate functional advanced materials, e.g., quantum dots to fabricate composite materials. Utilization of these compounds allows the advantages of both materials working in synergy to be harnessed to achieve superior performances in chemical sensing applications. ${ }^{60-62}$ To date, a few review articles have been reported, summarizing the sensing applications of MOFs in recent years. ${ }^{45-56}$ However, a significant number of MOFs have been explored for sensing applications in recent years, thus it is difficult to summarize most of the studies in a single review article. Furthermore, despite the aforementioned reviews on MOFbased sensing, we believe a focused review on the sensing of cationic species (both organic and inorganic) with MOFs will be a good addition to the literature. Thus, herein, our effort has been devoted to summarizing the sensing of cationic inorganic and organic species with the luminescent metalorganic frameworks reported in recent years.

\section{Luminescent metal-organic frameworks (LMOFs)}

MOFs have emerged as excellent materials for the fabrication of sensory probes in recent years. Luminescent MOFs (LMOFs) have been considered as an important class of MOFs, which have been utilized as sensory materials in various photonic studies, optoelectronics applications, etc. Functionalized LMOFs can be fabricated via both pre-synthetic and post-synthetic approaches, and can further be employed for sensing applications through the "turn-on" as well as "turn-off" phenomenon (Fig. 2). Moreover, the following origins of luminescence have been considered to play a fundamental role in MOFs (Fig. 3). ${ }^{63}$

\subsection{Organic ligand-based emission}

Organic linkers have been found to play a crucial role in the photoluminescence behaviour of LMOFs. Especially, it has been observed that $\pi$-electron-rich organic linkers contribute significantly in the emission profile of LMOFs in most cases. The reasoning behind the importance of $\pi$-electron-rich linkers has been found to be the ability of their organic struts to absorb light for excitation. Specifically, $\pi$-electron-rich organic moieties are known to absorb light, which later leads to radiative transition via fluorescence or phosphorescence.

Furthermore, in many cases the $\pi \rightarrow \pi^{*}$ transition, $\mathrm{n} \rightarrow \pi^{*}$ transition or both transitions have been considered as the 


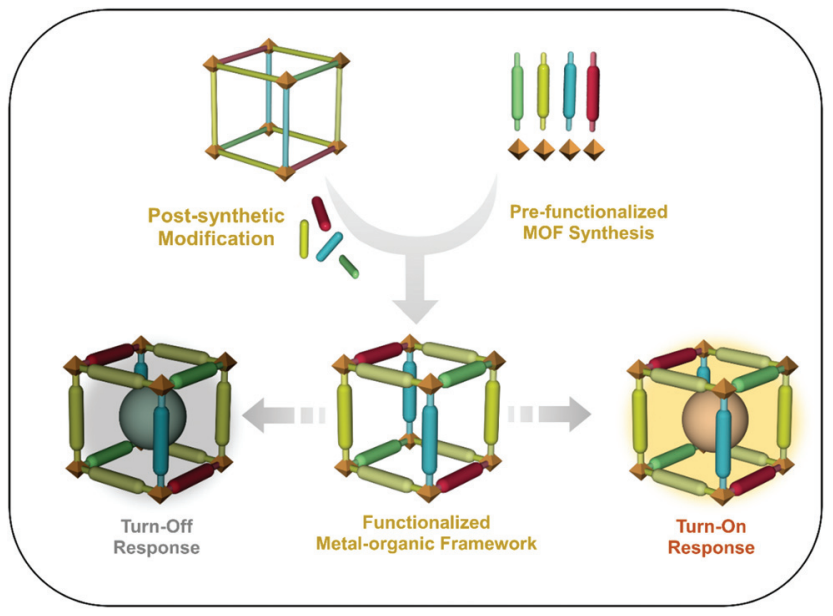

Fig. 2 Schematic representation of the development of luminescent metal-organic frameworks (LMOFs) for sensing applications via the "turn-on" and "turn-off" process.

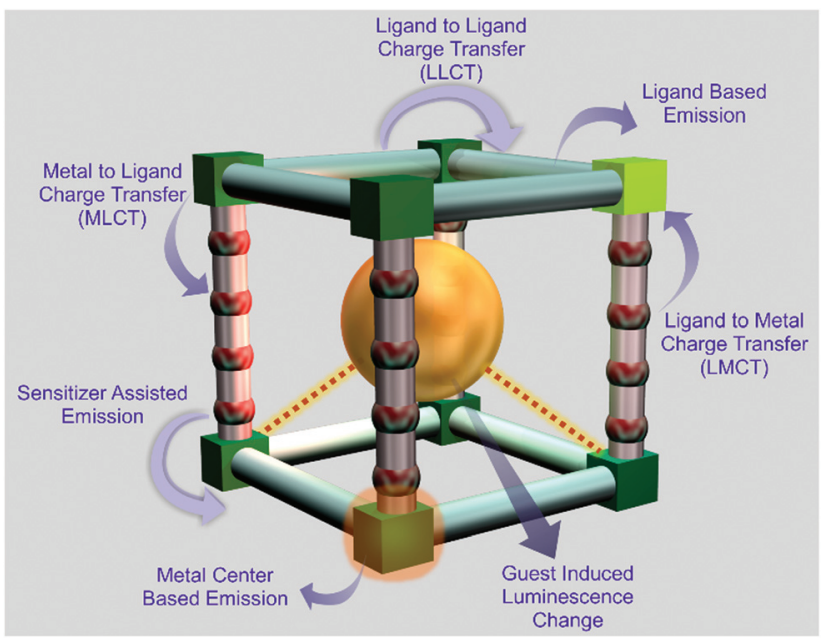

Fig. 3 Schematic representation of the plausible origin of luminescence in LMOFs.

origin of luminescence, where organic linkers have been found to play the key role. On the other hand, the metal nodes together with the linkers can contribute to the emission properties through different charge transitions. In this case, the luminescence property of LMOFs has been found to be governed by metal-to-ligand charge transfer (MLCT), ligand-tometal charge transfer (LMCT), inter-ligand charge transfer (ILCT), etc. Generally, MOFs based on metal ions with vacant outer d-orbitals (e.g. $\mathrm{Zr}(\mathrm{Iv})$ ) or completely occupied d-orbitals (Zn(II), Cd(II) etc.), have been observed to illustrate these phenomena.

\subsection{Metal ion/cluster-based emission}

Besides organic linker-based emission in LMOFs, metal ion- or metal cluster-based emission has been considered to be another important factor in LMOFs. In the case of metal node- centered luminescence in LMOFs, lanthanide-based MOFs are well known in the literature. Due to the forbidden $f-f$ transition according to the Laporte selection rule, generally, lanthanides offer very weak excitation together with very low quantum yield. Thus, to overcome this problem, there is a need for complexation between lanthanide metal ions and a sensitizer, which can absorb light and further transfer energy. In LMOFs, organic linkers have been found to act as efficient sensitizers, and this effect is termed the "antenna effect". Additionally, in case of lanthanides, the transitions occur from the f-orbitals, that are shielded by the 5s-orbitals and 5p-orbitals. As a result of this shielding, sharp and narrow emission profiles have been observed for lanthanides based LMOFs (since the f-orbitals remains undisturbed from the outer electronic environments). Besides this lanthanide metal nodebased emission, metal clusters in LMOFs have also led to excellent emissive features. In a recent report, Huang et al. reported a luminescent silver chalcogenide/chalcogenolate cluster-based MOF (viz., $\left[\left(\mathrm{Ag}_{12}\left(\mathrm{~S}^{t} \mathrm{Bu}\right)_{6}\left(\mathrm{CF}_{3} \mathrm{COO}\right)_{6}\left(\mathrm{CH}_{3} \mathrm{CN}\right)_{6}\right]\right.$. $\mathrm{CH}_{3} \mathrm{CN}$ ) with a quantum yield of $12.1 \%$ for the detection of oxygen and ethanol. ${ }^{64}$

\subsection{Guest or adsorbed lumophore-based emission}

Inclusion of luminescent guests or lumophores inside the networks of MOFs to fabricate luminescent MOFs is another excellent strategy explored in recent years. In this regard, various luminescent dye molecules, highly conjugated polyaromatic organic compounds, and various lanthanide metal ions have utilized as guests to fabricate LMOFs. These lumophores have been incorporated inside MOFs either via the adsorption technique or ion exchange technique. This strategy has been found to be an exciting path for the fabrication of desirable luminescent MOFs toward targeted applications.

\subsection{Exciplex formation-based emission}

The short lived homomeric and heteromeric species formed in the excited state are known as excimers or exciplex, respectively. Generally these species are very short lived and are known to dissociate in the ground state. Furthermore, excimers are the homomeric species formed in the excited state, while, exciplex are the heteromeric species formed in the excited state. For LMOFs, these species can be formed inside MOFs between organic linkers depending on their orientations in the excited state. On the other hand, adsorbed guest molecules can also contribute to the formation of this type of complex. Adsorbed guest molecules can form excimers or exciplex either via the interactions between the guests and organic linkers of MOFs or through the interactions between the absorbed guest molecules themselves. A detailed discussion regarding the origin of the luminescence in MOFs and luminescent MOFs has been documented in some recent reviews. Tremendous efforts have been devoted in recent years to the design and synthesis of functionalized LMOF-based sensors for the selective and sensitive detection of various toxic and hazardous cationic pollutants. Here, in the following sections 
we focused on summarizing the recent reports on the detection of cationic inorganic and organic species by MOFs.

\section{Recognition of cationic pollutants with MOFs}

\subsection{Recognition of inorganic cationic pollutants}

3.1.1 Sensing of heavy metals with MOFs. There is no widely accepted definition of a heavy metal, and thus it varies in the fields of physics, chemistry and biology. Chemists mainly classify heavy metals according to their density and properties. The heavy metals considered as toxic water pollutants are $\mathrm{Hg}^{2+}, \mathrm{Pb}^{2+}, \mathrm{Pd}^{2+}$, and $\mathrm{Cd}^{2+}$. The major sources of heavy metal water contamination are industrialisation and urbanisation. The presence of trace amounts of heavy metals in water bodies can cause serious health problems in humans and pose a threat to other ecosystems. The high solubility of these metal contaminants cause them to be widely distributed in various water bodies. Further, the adsorption of these metal contaminants by different living systems can lead to bioaccumulation in the food chain, and subsequently lethal consequences in humans. $\mathrm{Cd}^{2+}, \mathrm{Hg}^{2+}, \mathrm{As}^{3+}$, and $\mathrm{Pd}^{2+}$ have strong affinity for sulphur, and thus they usually bind with the - $\mathrm{SH}$ group of enzymes responsible for controlling the rate of metabolic reactions in the human body. Heavy metal poisoning can lead to several diseases, and some are extremely lethal to humans. Consequently, the United States (US) Environmental Protection Agency (EPA) has added heavy metals such as mercury, lead, cadmium, arsenic, and chromium to the priority pollutant list. ${ }^{65}$ Therefore, the detection of heavy metals is essential. Accordingly, MOF-based luminescent sensors have shown tremendous potential in the detection of these cation heavy metal pollutants in recent past years. Both MOFs and MOF-based composite materials have been employed for the selective sensing of heavy metal ions in the aqueous phase. In the following sections, a few representative literature reports are reviewed.

3.1.1 a Mercury $(\mathrm{Hg})$ sensing. Among the metal contaminants, mercury $(\mathrm{Hg})$ is considered as one of the most toxic and hazardous elements. Pollution due to mercury ions $\left(\mathrm{Hg}^{2+}\right)$ has been found to be widespread. In addition, several natural and anthropogenic sources have been considered as the cause of mercury contamination in different water bodies, soil, etc. Furthermore, the bioaccumulation of mercury in various food chains has been found to be facilitated by the conversion the inorganic $\mathrm{Hg}^{2+}$ ion to methylmercury $\left(\mathrm{MeHg}^{+}\right)$by different bacteria. This type of bioaccumulation of mercury in the food chain has led to several fatal diseases in humans. Thus, because of these ill effects, the US EPA has set the permissible level of $\mathrm{Hg}^{2+}$ ions in water to as low as $2 \mathrm{ppb}^{66}$ Several MOFbased sensory probes have been used for the detection of this toxic water pollutant. In a recent study, our group reported an efficient chemosensor for $\mathrm{Hg}^{2+}$ ions in water medium based on a functionalized MOF, viz. UiO-66@butyne (isoreticular with UiO-66), featuring an active butyne functionality
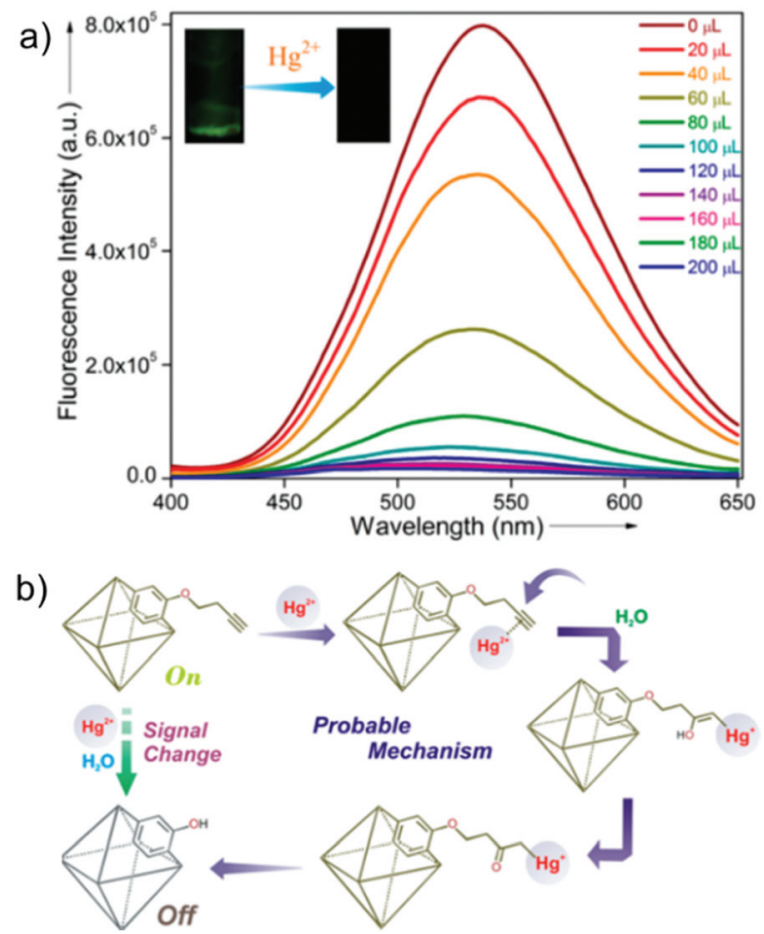

Fig. 4 (a) Fluorescence quenching of UiO-66abutyne upon the addition of $\mathrm{Hg}^{2+}$ ion solution in water. (b) Schematic representation for the plausible mechanism of $\mathrm{Hg}^{2+}$ ion sensing with UiO-66@abutyne. Reproduced with permission from ref. 66. Copyright 2018, American Chemical Society.

(Fig. 4a) ${ }^{66}$ Interestingly, the luminescence quenching process involved an oxymercuration reaction-based chemodosimetric pathway with high sensitivity $(\mathrm{LOD}=10.9 \mathrm{~nm})$ and good selectivity in the presence of interfering metal cations (Fig. 4b). The reaction-based mechanism was also validated thorough a control experiment. In another report, Chen and co-workers were able to develop for the first time a lanthanide coordination polymer nanoparticle (CPNP) containing adenine as a co-linker together with dipicolinic acid (DPA) connected through $\mathrm{Tb}^{3+}$ metal nodes (Ad/Tb/DPA CPNPs). ${ }^{67}$ The pristine CPNP behaved as a weakly fluorescent material, which can be enhanced significantly with the introduction of $\mathrm{Hg}^{2+}$ ions. The authors stated that the suppressed fluorescence was a consequence of the photoinduced electron transfer (PET) from the adenine nucleobase to the DPA co-linker, which is largely inhibited in presence of $\mathrm{Hg}^{2+}$ ions by virtue of the coordination between adenine and $\mathrm{Hg}^{2+}$ ions. The "turn-on" response was found to be very selective and sensitive (LOD = $0.2 \mathrm{nM}$ ). It is worth mentioning that this work represents the first example of a coordination polymer-based $\mathrm{Hg}^{2+}$ sensor operating via the PET mechanism.

Moreover, Yang and co-workers contributed by developing a strongly fluorescent LnMOF film, enabling convenient sensing of $\mathrm{Hg}^{2+}$ ions in DMF. ${ }^{68}$ The thin films were fabricated via electrodeposition followed by a solvothermal procedure using a luminescent LnMOF, $\left\{\left[\mathrm{Eu}_{2}(\mathrm{bqdc})_{3}\left(\mathrm{H}_{2} \mathrm{O}\right)(\mathrm{DMF})_{3}\right] \cdot 0.5 \mathrm{DMF} \cdot \mathrm{H}_{2} \mathrm{O}\right\}_{n}$ 
(1, bqdc: 2,2'-biquinoline-4,4' dicarboxylate). The LnMOF thin film was found to be extremely sensitive for the detection of $\mathrm{Hg}^{2+}\left(1 \times 10^{-8} \mathrm{M}\right)$ via luminescence quenching, while a portable sensor device was also fabricated for the convenient detection of $\mathrm{Hg}^{2+}$ ions. Recently, Wen and co-workers reported two new MOFs with decorated amine functionality, viz. [ $\mathrm{Zn}(2-$ $\mathrm{NH}_{2}$ bdc)(bibp) $]_{n} \quad\left[1 ; \quad 2-\mathrm{NH}_{2}\right.$ bdc: 2-amino-1,4-benzenedicarboxylic acid and bibp: 4,4'-bis(imidazol-1-ylmethyl)-biphenyl] and $\left[\mathrm{Cd}\left(2-\mathrm{NH}_{2} \mathrm{bdc}\right)(\mathrm{tib}) \cdot 4 \mathrm{H}_{2} \mathrm{O} \cdot 0.5 \mathrm{DMA}\right]_{n}[2$; tib: 1,3,5-tris $(1-\mathrm{imi}-$ dazolyl)benzene and DMA: dimethylacetamide], acting as a "turn-off" fluorescent sensor for $\mathrm{Hg}$ (II) and $\mathrm{Cr}(\mathrm{VI}) .{ }^{69}$ Both MOFs were efficient sensors for $\mathrm{Hg}^{2+}$ with 1 having a $K_{\mathrm{sv}}$ value of $4550 \mathrm{M}^{-1}$, while 2 exhibited an LOD value of $4.2 \times 10^{-8} \mathrm{M}$ in aqueous solution. Additionally, a complexation-driven quenching mechanism was also proposed as the sensing mechanism.

Similarly, Wang and co-workers documented a new CdMOF, $\left\{\left[\mathrm{Cd}_{1.5}\left(\mathrm{C}_{18} \mathrm{H}_{10} \mathrm{O}_{10}\right)\right] \cdot\left(\mathrm{H}_{3} \mathrm{O}\right)\left(\mathrm{H}_{2} \mathrm{O}\right)_{3}\right\}_{n}$ (Cd-EDDA) (EDDA: [ethane-1,2 diylbis(oxy)]diisophthalic acid), for sensing $\mathrm{Hg}^{2+}$ ions in water. ${ }^{70} \mathrm{Cd}$-EDDA was found to detect $\mathrm{Hg}^{2+}$ ions selectively $\left(K_{\mathrm{sv}}=4.3 \times 10^{3} \mathrm{M}^{-1}\right)$ in the presence of competing analytes with acceptable sensitivity (LOD $=2 \mathrm{nM}$ ) in a highly preferable ratiometric manner.

Notably, this work illustrated the first ratiometric $\mathrm{Hg}^{2+}$ sensor in water. The sensing was reported to proceed via $\mathrm{Hg}^{2+}$-induced framework collapse. Recently, Li and co-workers reported a series of LMOFs, namely $\mathrm{Zn}_{2}$ (ofdc) $)_{2}$ (tppe) (LMOF-261), $\mathrm{Zn}_{2}$ (hfdc) $)_{2}$ (tppe) (LMOF-262), $\mathrm{Zn}_{2}\left(\text { dbtdcO }_{2}\right)_{2}$ (tppe) (LMOF-263) (ofdc: 9-oxo-9H-fluorene-2,7-dicarboxylic acid, hfdc: $9 H$-fluorene-2,7-dicarboxylic acid, and $\mathrm{dbtdcO}_{2}$ : dibenzo $[b, d]$ thiophene-3,7-dicarboxylic acid-5,5-dioxide), among which, LMOF-263 exhibited remarkable potential for the simultaneous detection and scavenging of heavy metal ions $\left(\mathrm{Hg}^{2+}\right.$ and $\mathrm{Pb}^{2+}$ ) in water medium (Fig. 5a). ${ }^{71}$ Equipped with a functional thio unit, LMOF-263 behaved as an exceptional chemosensor for $\mathrm{Hg}^{2+}$ ions via fluorescence quenching with a high $K_{\text {sv }}$ value (459 $446 \mathrm{M}^{-1}$ ) and low LOD (3.3 ppb). Furthermore, the probe was found to be reusable, and the interaction of $\mathrm{Hg}^{2+}$ ions with the sulfone unit was established as the mechanism for luminescence quenching (Fig. 5b).

3.1.1b Palladium $(P d)$ sensing. Palladium $(\mathrm{Pd})$ is a rare transition element and it has wide range of applications in diverse fields. It has been widely used as a heterogeneous catalyst, also in electronics, dental materials, jewellery, etc. However, a very low amount of Pd has adverse effects on human health. $\mathrm{Pd}^{2+}$ can bind with DNA, RNA, amino acids and other macromolecules such as vitamin B6, resulting in several problems. Also it causes cytotoxicity and allergies in the human body. Thus, to manage this hazard, environment agencies have specified the intake limit of $\mathrm{Pd}^{2+}$, where the maximum dietary intake of palladium per day per person is limited to less than $1.5-15 \mu \mathrm{g}$ and the threshold in drugs is $5-10 \mathrm{ppm}$. In one of the early reports, $\mathrm{Xu}$ and co-workers presented a stand out example of $\mathrm{Pd}(\mathrm{II})$ detection in single crystals with a newly synthesized MOF, ASMOF-5 $\left[\mathrm{Zn}_{4} \mathrm{O} \cdot(\mathrm{L})_{3} \cdot(\mathrm{DEF})_{2} \cdot\left(\mathrm{H}_{2} \mathrm{O}\right)_{2}\right]$ (L: 2,5dithioalloxyterephthalic acid) (Fig. 6a). ${ }^{72}$ The as-synthesized MOF bearing both alkene and thioether functionality was

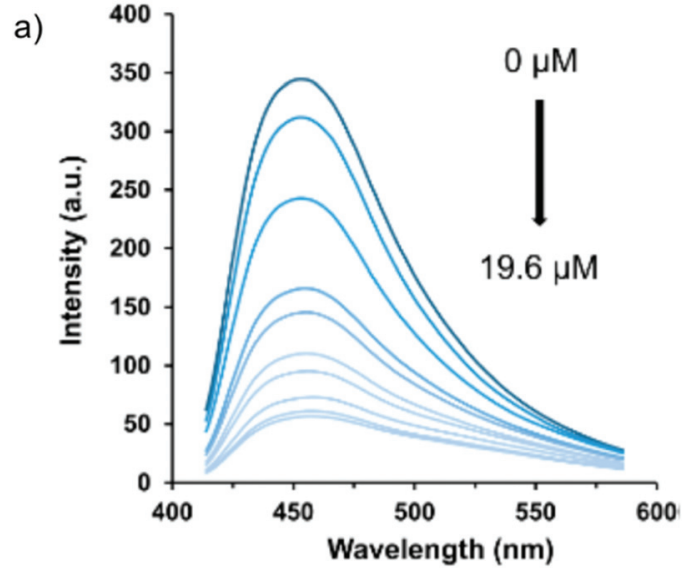

b)

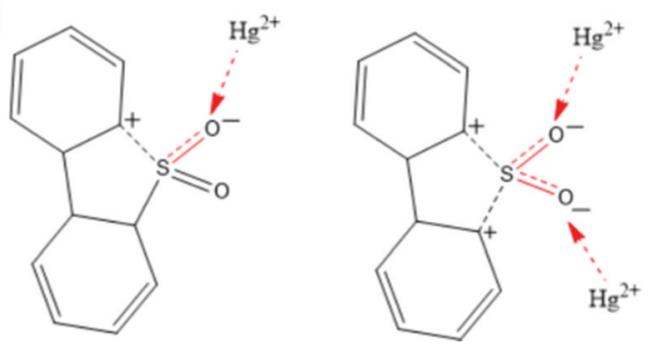

Fig. 5 (a) Quenching of the luminescence profile of LMOF-263 after the incremental addition of an $\mathrm{Hg}^{2+}$ ion solution. (b) Interaction of $\mathrm{Hg}^{2+}$ ions with the sulfone functional group. Reproduced with permission from ref. 71. Copyright 2016, American Chemical Society.

found to be isoreticular with MOF-5 and displayed a selective colorimetric response (light yellow to orange/brick red) through a wide range of $\mathrm{Pd}$ concentrations (5-1500 ppm) (Fig. 6b). Notably, other noble metal ions and transition metals did not induce any color change at a lower concentration range $(50 \mathrm{ppm})$, while at higher concentrations (>1000 ppm) the brownish-grey color generated by other metals could be easily discriminated from the orange-red color induced by $\mathrm{Pd}^{2+}$ ions. In addition, an equimolar mixture of interfering metal ions did not influence the colorimetric response (except $\mathrm{Ru}(\mathrm{III})$ ). Finally, to verify the role of the alkene group in ASMOF-5, the authors investigated the detection of $\mathrm{Pd}^{2+}$ using the reported SESMOF-5 (with a linker side chain of $\mathrm{CH}_{3} \mathrm{SCH}_{2} \mathrm{CH}_{2} \mathrm{~S}^{-}$) and MOF-5 (non-functionalized), and observed an unnoticeable color change for SESMOF-5, while MOF-5 did not show any color change, highlighting the role of the alkene functionality in ASMOF-5.

Furthermore, Konar and co-workers reported a new luminescent $\quad$ MOF, $\left\{\left[\mathrm{Zn}\left(\mathrm{C}_{34} \mathrm{H}_{18} \mathrm{O}_{8}\right)_{0.5}\left(\mathrm{C}_{20} \mathrm{~N}_{2} \mathrm{H}_{16}\right)_{0.5}\right] \cdot[0.5\right.$ $\left.\left.\left(\mathrm{C}_{20} \mathrm{~N}_{2} \mathrm{H}_{16}\right) \cdot 2 \mathrm{H}_{2} \mathrm{O}\right]\right\}_{n}$ (1), built from a conjugated, rigid bpeb linker (bpeb: 1,4-bis[2-(4-pyridyl)ethenyl]benzene) combined with the $\pi$-electron-rich $\mathrm{H}_{4}$ tcpb (1,2,4,5-tetrakis(4-carboxyphenyl)benzene) for the selective detection of $\mathrm{Pd}^{2+}$ ions and picric acid. ${ }^{73}$ The crystal structure of 1 revealed the presence of the bpeb linker-bearing alkene moiety both inside the pores and on the pore walls, which was further employed for the detection of $\mathrm{Pd}^{2+}$ in DMF solution. The high efficiency of $\mathrm{Pd}^{2+}$ detec- 
a)

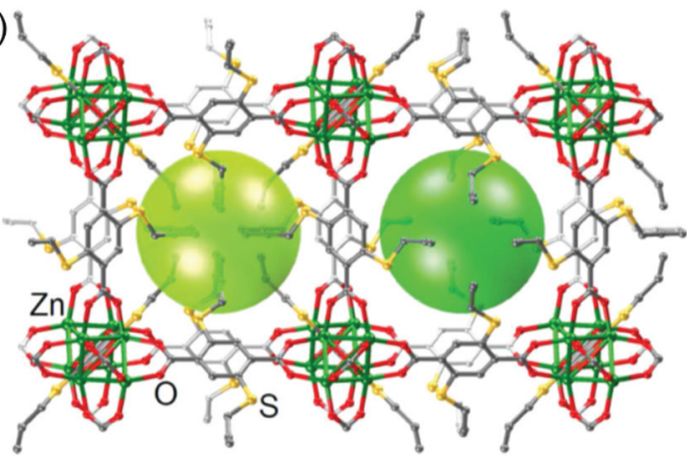

b)
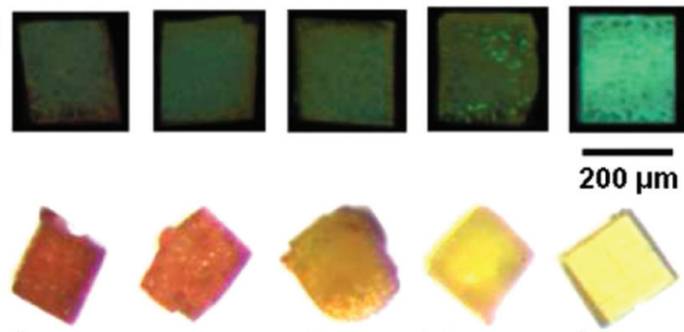

$10 \mathrm{ppm}$
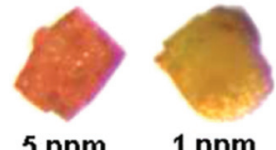

$1 \mathrm{ppm}$

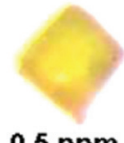

$200 \mu \mathrm{m}$

Fig. 6 (a) Single-crystal structure of ASMOF-5. (b) Images of ASMOF-5 crystals under UV-radiation ( $\lambda=365 \mathrm{~nm}$ ) (top) and under normal light (bottom) before and after immersion in $\mathrm{Pd}\left(\mathrm{CH}_{3} \mathrm{CN}\right)_{2} \mathrm{Cl}_{2}$ solution in $\mathrm{CH}_{2} \mathrm{Cl}_{2}$ at various concentrations at $80^{\circ} \mathrm{C}$ with a duration of immersion of: $1 \mathrm{~h}$ (top) and $4 \mathrm{~h}$ (bottom). Reproduced with permission from ref. 72. Copyright 2013, American Chemical Society.

tion was also supported by its high $K_{\mathrm{sv}}$ value of $3.6 \times 10^{4} \mathrm{M}^{-1}$ and low limit of detection (0.03 ppm). Additionally, an interaction-based mechanism between the alkene moiety and $\mathrm{Pd}^{2+}$ ions was suggested for the luminescence quenching. In another contribution, Suresh and co-workers demonstrated the potential of LMOFs toward $\mathrm{Pd}^{2+}$ sensing in aqueous medium by synthesizing two 3D isostructural MOFs having the general formula of $\left\{[\mathrm{M}(\mathrm{ATA})(\mathrm{L})] \cdot x \mathrm{H}_{2} \mathrm{O}\right\}_{n}(\mathrm{M}=\mathrm{Zn}$ for LMOF1 and $\mathrm{Cd}$ for LMOF2) ${ }^{74}$ The two MOFs, composed via a mixed ligand strategy using 4-pyridyl carboxaldehyde isonicotinoylhydrazone $(\mathrm{L})$ and 2-aminoterephthalic acid $\left(\mathrm{H}_{2} \mathrm{ATA}\right)$, were able to detect $\mathrm{Pd}^{2+}$ (also $\mathrm{Fe}^{3+}$ ) via luminescence quenching. The efficacy of both probes was very good, as evident from their high $K_{\mathrm{sv}}$ values $\left(4.182 \times 10^{4} \mathrm{M}^{-1}\right.$ for LMOF1 and $7.872 \times 10^{4}$ $\mathrm{M}^{-1}$ for LMOF2) and impressive LOD (35 ppb for LMOF1 and $18 \mathrm{ppb}$ for LMOF2). Furthermore, a donor-acceptor based mechanism was proposed, involving the amine and amide functionality of the linkers. Notably, this was the first report illustrating the aqueous phase recognition of $\mathrm{Pd}^{2+}$ ions employing LMOFs.

Helal and co-workers reported an LnMOF, namely KFUPM-3, constructed from $\mathrm{Eu}^{3+}$ metal nodes and a 2,5-bis (allyloxy)terephthalic acid $\left(\mathrm{H}_{2} \mathrm{BAT}\right)$ linker as a competent chemosensor for $\mathrm{Pd}^{2+}$ ions in water (Fig. 7a). ${ }^{75}$ KFUPM-3 was found to selectively sense $\mathrm{Pd}^{2+}$ ions even in the presence of interfering metal cations, which had a high $K_{\mathrm{sv}}$ value of $7.8 \times$ $10^{4}$ and very low LOD of $44 \mathrm{ppb}$ (Fig. 7b). Also, this chemosensor was reusable for at least 4 cycles, and a coordinative
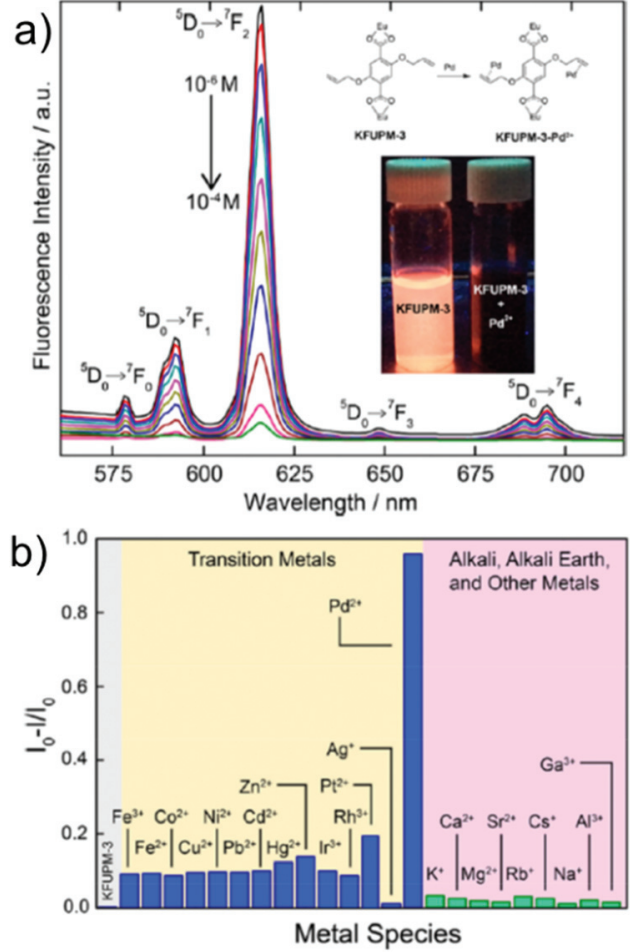

Fig. 7 (a) Changes in the luminescence profile of KFUPM-3 upon the incremental addition of $\mathrm{PdCl}_{2}$ in water (inset: mechanism of sensing and fluorescence quenching upon the addition of $\mathrm{Pd}^{2+}$ ). (b) Selectivity study of the probe for $\mathrm{Pd}^{2+}$ ions. Reproduced with permission from ref. 75 . Copyright 2019, American Chemical Society.

interaction-based mechanism between the functional allyloxy unit and $\mathrm{Pd}^{2+}$ ions was proposed for the luminescence quenching. Recently, Hmadeh and co-workers developed 2D layered MOF nanosheets (AUBM-6-NS) based on $\mathrm{Cu}^{2+}$ and an easily available isonicotinic acid (INA) linker as an effective "turnoff" sensor toward $\mathrm{Pd}^{2+}$ ions. ${ }^{76}$ Few-layer AUBM-6-NS was obtained via the liquid-assisted exfoliation of the synthesized 2D layered AUBM-6 MOF. Further, AUBM-6-NS displayed efficient $\mathrm{Pd}^{2+}$ detection with high selectivity $\left(K_{\mathrm{sv}}=1.6 \times 10^{4}\right.$ $\mathrm{M}^{-1}$ ) and sensitivity (LOD $\left.=0.02 \mathrm{ppm}\right)$.

3.1.1c Lead $(P b)$ sensing. Besides mercury, lead is another well-recognized hazardous heavy metal, which is known for its high toxicity to living systems. Lead poisoning can lead to gastrointestinal tract alterations, hemotoxic effects, reproductive dysfunction, nephropathies, etc. Furthermore, acute poising of lead can cause diarrhea, weight loss, inflammation of the brain, etc. Especially, for children, lead poisoning is very dangerous as it can cause slower neurological and decrease in intelligence even at a level as low as $10 \mathrm{ppb}$ lead. $^{77}$ Consequently, the detection of lead in aqueous medium has emerged as one of the very important topics of research in recent years. Liu and co-workers reported a new $2 \mathrm{D}$ green luminescent Ln-MOF, $\left[\mathrm{Tb}(\mathrm{L})\left(\mathrm{H}_{2} \mathrm{O}\right)_{5}\right]_{n}$ solvent (Tb-MOF, $\mathrm{H}_{2} \mathrm{~L}$ : $3,5^{-}$ dicarboxyphenol anion ligand), for the effective sensing of toxic $\mathrm{Pb}^{2+}$ ions in water. The MOF showed outstanding 
efficiency toward the detection of $\mathrm{Pb}^{2+}$ in the presence of other metal cations with great selectivity $\left(K_{\mathrm{sv}}=1.75 \times 10^{4} \mathrm{M}^{-1}\right)$ and appreciable sensitivity (LOD $\left.=10^{-7} \mathrm{M}\right) \cdot{ }^{78}$ Additionally, the authors probed the mechanism of the luminescence quenching, and a crystal film was also fabricated through pressing technology aimed at the practical applicability of the sensor. Besides this photoluminescence-based sensing of $\mathrm{Pb}^{2+}$ ions, a few electrochemical sensors have also been explored based on MOFs. ${ }^{79-81}$

3.1.1d Cadmium (Cd) sensing. Cadmium is considered as one of the most toxic and hazardous elements among the heavy metals. Even at a very low concentration, it can lead to various health disorders. Cadmium poisoning can lead to lethal diseases such as cancer, diabetes, kidney and liver failure, and several heart diseases. Also, itai-itai disease is one of the well-known diseases caused by exposure to cadmium. Consequently, the United States (US) EPA has set the permissible level of Cd in water to $5 \mathrm{ppb}^{82}$ On the other hand, according to the World Health Organization (WHO), the lower limit of cadmium in water should be $3 \mathrm{ppb}$. Thus, the sensing of $\mathrm{Cd}^{2+}$ ions in water has become essential. However, although a few MOF-based electrochemical sensors have been reported for $\mathrm{Cd}^{2+}$ ions, MOF-based photoluminescent sensors are very rare. ${ }^{82,83}$ Recently, Yan and co-workers adopted a post-synthetic modification strategy to anchor $\mathrm{Eu}^{3+}$ cations in UiO-66 $(\mathrm{Zr})-(\mathrm{COOH})_{2}$ to act as a "turn-on" sensor toward $\mathrm{Cd}^{2+}$ ions in water (Fig. 8a). ${ }^{84}$ The well-known UiO-66(Zr)-(COOH $)_{2}$ was synthesized using $\mathrm{Zr}(\mathrm{Iv})$ and $\mathrm{H}_{4}$ btec as the linker with a molecular formula of $\left[\mathrm{Zr}_{6} \mathrm{O}_{4}(\mathrm{OH})_{4}\left(\mathrm{H}_{2} \mathrm{btec}\right)_{6}\right]$ (where, $\mathrm{H}_{4}$ btec: 1,2,4,5-benzenetetracarboxylic acid). The post-synthetic modification was carried out by keeping the MOF in an aqueous solution of $\mathrm{EuCl}_{3}$ to incorporate $\mathrm{Eu}^{3+}$ ions. After the incorporation of $\mathrm{Eu}^{3+}$ inside UiO-66(Zr)- $(\mathrm{COOH})_{2}$, a red emission was observed from the MOF-composite. Additionally, five emission peaks for the material were observed at $578 \mathrm{~nm}, 591 \mathrm{~nm}, 614 \mathrm{~nm}, 650 \mathrm{~nm}$ and $695 \mathrm{~nm}$, corresponding to the ${ }^{5} \mathrm{D}_{0} \rightarrow{ }^{7} \mathrm{~F}_{0},{ }^{5} \mathrm{D}_{0} \rightarrow{ }^{7} \mathrm{~F}_{1},{ }^{5} \mathrm{D}_{0} \rightarrow$ ${ }^{7} \mathrm{~F}_{2},{ }^{5} \mathrm{D}_{0} \rightarrow{ }^{7} \mathrm{~F}_{3}$ and ${ }^{5} \mathrm{D}_{0} \rightarrow{ }^{7} \mathrm{~F}_{4}$ transitions, respectively. This modified MOF showed a long lifetime of $341.7 \mu$ s together with a high quantum yield of $20.5 \%$. Further, this MOF was employed for sensing $\mathrm{Cd}^{2+}$ in aqueous medium. An enhancement in fluorescence (8-fold) was observed exclusively for $\mathrm{Cd}^{2+}$ ions, while concurrently in the presence of other metal ions, the MOF was observed to be inactive, which indicated the selectivity of the probe. Moreover, the detection limit of the probe was determined to be $0.06 \mu \mathrm{M}$ toward the analyte (Fig. 8b). Also, the proposed mechanism is based on $\mathrm{Cd}^{2+}$ interacting with the Lewis basic $-\mathrm{COOH}$ group, which in turn facilitates the energy transfer from the ligand to $\mathrm{Eu}^{3+}$ cations.

3.1.1e Chromium (Cr) sensing. Chromium is well known for its highly carcinogenic and mutagenic nature. Although a trace amount of $\mathrm{Cr}$ (III) is required in the human body, its excess can lead to mutations and malignant cells. ${ }^{85}$ Recently Gu, Liu and co-workers reported an $\mathrm{Zn}$-based MOF (namely, $\mathrm{NH}_{2}-\mathrm{Zn}$-MOF) with the molecular formula of $\left[\mathrm{Zn}_{2}(\mathrm{TPOM})\left(\mathrm{NH}_{2}-\mathrm{BDC}\right)_{2}\right] \cdot 4 \mathrm{H}_{2} \mathrm{O}$ for the sensing of $\mathrm{Cr}^{3+}$ ions. ${ }^{86}$ An increment in the emission profile of the MOF together with a red shift was observed with
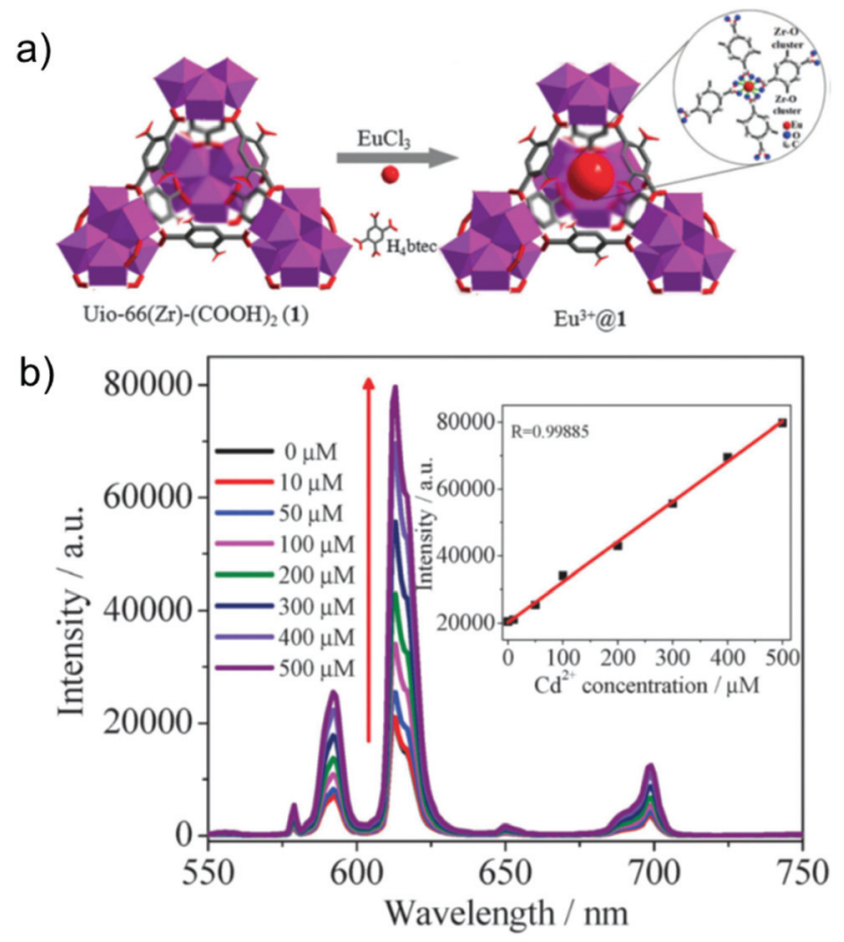

Fig. 8 (a) Synthetic representative structure of $\mathrm{Eu}^{3+} \mathrm{aMOF}$. (b) Fluorescence intensity change of $\mathrm{Eu}^{3+} \mathrm{a1}$ upon the addition of $\mathrm{Cd}^{2+}$ ions (inset: linear relationship of the fluorescence intensity of the probe enhanced by $\mathrm{Cd}^{2+}$ ions). Reproduced with permission from ref. 84 . Copyright 2015, Royal Society of Chemistry.

the incremental addition of $\mathrm{Cr}^{3+}$ ions. This probe was found to be highly selective and its limit of detection was calculated to be $4.9 \mu \mathrm{M}$. This enhancement in the photoluminescence profile was explained in terms of the chelation of $\mathrm{Cr}^{3+}$ ions by the oxygen atom of the carboxylate group and nitrogen atom of the amine group, leading to a hexatomic ring, which further led to rigidity in the structure and more conjugation. This probe was further employed for the sensing of $\mathrm{Fe}^{3+}$ ions. Another Zn-MOF with the formula of $\left[\mathrm{Zn}(\mathrm{L})\left(\mathrm{H}_{2} \mathrm{O}\right)\right] \cdot \mathrm{H}_{2} \mathrm{O}\left(\mathrm{H}_{2} \mathrm{~L}\right.$ : 5-(2-methylpyridin-4-yl)isophthalic acid) was employed by Wang and co-workers for the sensing of $\mathrm{Cr}^{3+}$ ions. ${ }^{87} \mathrm{An}$ emission peak at $426 \mathrm{~nm}$ was observed for the MOF when it was excited at $346 \mathrm{~nm}$ in water medium. Furthermore, this MOF was found to be stable in the $\mathrm{pH}$ range of 2-11 and its luminescence remained unchanged in various $\mathrm{pH}$ media. A drastic quenching in the luminescence profile of the MOF was obtained upon the addition of $\mathrm{Cr}^{3+}$ ions. This rapid quenching was also reflected in the quenching constant, where a high $K_{\mathrm{sv}}$ of $2.03 \times 10^{4} \mathrm{M}^{-1}$ was observed for the probe. In addition to the high quenching constant, the low detection limit of the probe $(2.44 \mu \mathrm{M})$ also depicted the efficiency of the compound. The reversibility of the MOF was further checked, and it was found that even after four cycles, the efficiency of the probe remained unaltered.

Furthermore, Liu and co-workers reported a red emissive Eu-MOF for the quantitative sensing of $\mathrm{Cr}^{3+}$ ions in 
a)

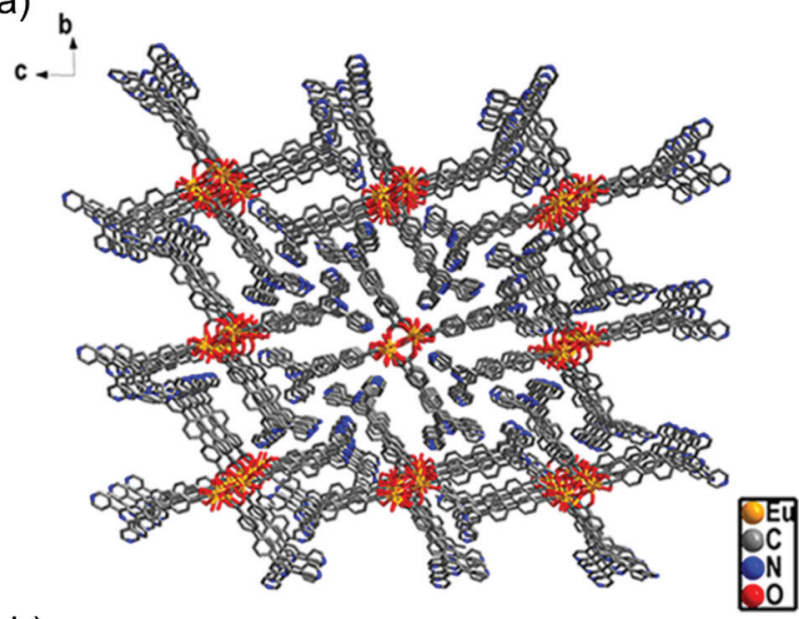

b)

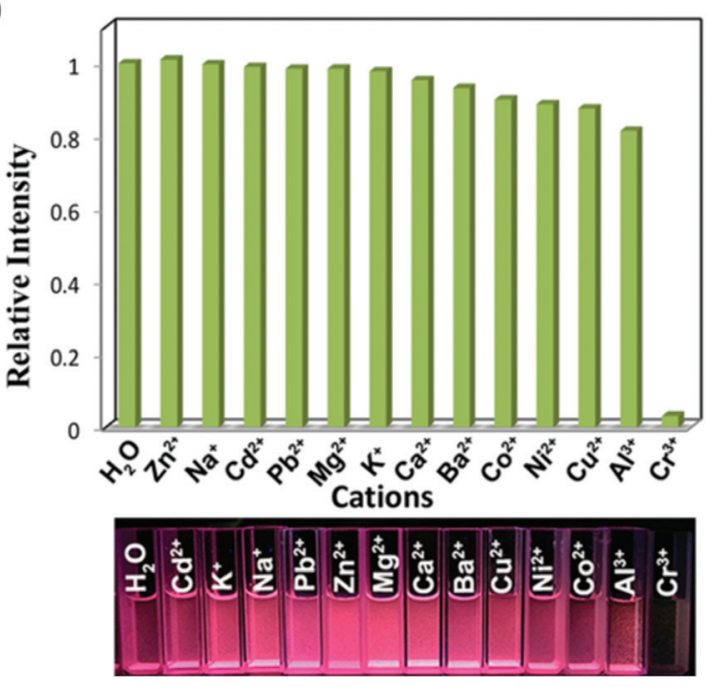

Fig. 9 (a) Crystal structure of the MOF. (b) Bar diagram and images representing the selectivity of the probe for $\mathrm{Cr}^{3+}$ ions. Reproduced with permission from ref. 88. Copyright 2017, American Chemical Society.

aqueous medium. This MOF with the formula of $\left[\mathrm{Eu}_{2}(\mathrm{tpbpc})_{4} \cdot \mathrm{CO}_{3} \cdot 4 \mathrm{H}_{2} \mathrm{O}\right] \cdot \mathrm{DMF} \cdot$ solvent (Htpbpc: $4^{\prime}-\left[4,2^{\prime} ; 6^{\prime}, 4^{\prime \prime}\right]-$ terpyridin-4'-yl-biphenyl-4-carboxylic acid) was synthesized under solvothermal conditions. ${ }^{88}$ The one-dimensional chains of the metal-linker complex were found to form a three dimensional supramolecular structure via hydrogen bonding (Fig. 9a). This compound was observed to be stable in water medium and at various $\mathrm{pH}$. Moreover, a rapid quenching of the red emission of the Eu-MOF was observed upon the addition of $\mathrm{Cr}^{3+}$ ions. The detection limit of the probe for the targeted analyte was calculated to be $3.64 \mathrm{ppm}$ with a quenching constant of $5.14 \times 10^{2} \mathrm{M}^{-1}$. Additionally, this probe was found to be very selective for the $\mathrm{Cr}^{3+}$ ions over other concurrent metal cations (Fig. 9b). Also, besides these aforementioned reports, there are few other reports on the sensing of $\mathrm{Cr}^{3+}$ ions with MOFs. ${ }^{89-94}$

\subsubsection{Sensing of other metals with MOFs}

3.1.2a Iron ( $\mathrm{Fe}$ ) sensing. It is well recognized that iron (Fe), apart from being the highest prevailing transition metal in humans (3-5 $\mathrm{g}$ for average adult human), is an indispensable and ubiquitous metal in biological organisms. It is responsible for crucial biological processes such as metabolism, electron and oxygen transport and the synthesis of DNA. Both insufficient and excess iron disrupts the regulation of iron and can cause serious health disorders including Alzheimer's disease, anemia, diabetes, and hemochromatosis. Additionally, the permissible limit for iron determined by the World Health Organization (WHO) is $1-3 \mathrm{ppm}$ for drinking anaerobic well water. ${ }^{95}$ Hence, it is imperative to develop competent sensors for the detection of $\mathrm{Fe}^{3+}$ and $\mathrm{Fe}^{2+}$ in trace amounts. Accordingly, a considerable amount of research has performed toward the development of new optical sensors featuring superior sensitivity, rapid response and improved selectivity. In this direction, luminescent MOFs (LMOFs) have shown remarkable potential for the fabrication of convenient, cost-effective and efficient probes. In this section, some of the important and recent results are discussed.

Recently, Zang and co-workers reported a new porous 3D lanthanide MOF, $\quad\left\{\left[\mathrm{Tb}_{4}(\mathrm{OH})_{4}(\mathrm{DSOA})_{2}\left(\mathrm{H}_{2} \mathrm{O}\right)_{8}\right] \cdot\left(\mathrm{H}_{2} \mathrm{O}\right)_{8}\right\}_{n} \quad$ (TbDSOA), built from a sulfonate-carboxylate-based linker, which can selectively detect $\mathrm{Fe}^{3+}$ ions. ${ }^{96}$ The tetranuclear $\mathrm{Tb}$ clusters sensitized by the ligand contributed to the characteristic green luminescence of the $\mathrm{Tb}$ (III) ions, while the uncoordinated oxygen atoms from the sulfonate groups served as basic functional sites for the selective sensing of $\mathrm{Fe}^{3+}$ ions via luminescence quenching. Additionally, the Tb-DSOA MOF showed a selective "turn-off" response toward $\mathrm{Fe}^{3+}$ even in the presence of several interfering metal cations with a $K_{\text {sv }}$ value of 3543 $\mathrm{M}^{-1}$. The authors also studied the probable working mechanism and suggested that the interaction between $\mathrm{Fe}^{3+}$ ions with the basic site within the pores is responsible for the luminescence quenching.

Broadening the scope of LMOFs in $\mathrm{Fe}^{3+}$ sensing Qian, Cui and co-workers reported the first few-layer 2D MOF nanosheets, NTU-9-NS $\mathrm{Ti}_{2}(\mathrm{HDOBDC})_{2}\left(\mathrm{H}_{2} \mathrm{DOBDC}\right)\left(\mathrm{H}_{2} \mathrm{DOBDC}\right.$ : 2,5-dihydroxyterephthalic acid; NTU: Nanyang Technological University), crafted via a top-down delamination approach. ${ }^{97}$ The exfoliated highly dispersive 2D nanosheets in synergy with greater accessible active sites exhibited exceptional luminescent sensing of $\mathrm{Fe}^{3+}$ ions with an ultrafast response time and outstanding detection limit $(0.45 \mu \mathrm{M})$ and high selectivity. In another report, Zheng and co-workers took advantage of the highly luminescent nature of lanthanide MOFs (Ln-MOFs) and synthesized two isostructural MOFs, namely $\{[\mathrm{Eu}(\mathrm{L})$ $\left.\left.(\mathrm{BPDC})_{1 / 2}\left(\mathrm{NO}_{3}\right)\right] \cdot \mathrm{H}_{3} \mathrm{O}\right\}_{n}(1)$ and $\left\{\left[\mathrm{Tb}(\mathrm{L})(\mathrm{BPDC})_{1 / 2}\left(\mathrm{NO}_{3}\right)\right] \cdot \mathrm{H}_{3} \mathrm{O}\right\}_{n}$ (2), which were further employed for the detection of in $\mathrm{Fe}^{3+} .{ }^{98}$ The as-synthesized 3D MOFs featured a 2-fold interpenetration with a free basic pyridine moiety available for the selective and sensitive recognition of $\mathrm{Fe}^{3+}$ ions in DMF medium. Moreover, both MOFs had good $K_{\text {sv }}$ values $\left(5.16 \times 10^{4} \mathrm{M}^{-1}\right.$ for 1 and 4.30 $\times 10^{4} \mathrm{M}^{-1}$ for 2) with $\mathrm{Fe}^{3+}$ and the probable mechanism for fluorescence quenching was also discussed. Furthermore, Mandal and co-workers extended the library of Ln-MOF sensors by reporting a new Eu-based MOF, $\left\{\mathrm{Eu}_{2} \mathrm{~L}_{3}(\mathrm{DMF})\right\} \cdot 2 \mathrm{DMF} \quad(\mathrm{L}: \quad$ (6-[1-(4-carboxyphenyl)-1H-1,2,3- 
triazol-4-yl]nicotinic acid), which was employed for the selective sensing of $\mathrm{Fe}^{3+} \cdot{ }^{99}$ The sensitivity was found to be quite $\operatorname{good}(\mathrm{LOD}=6.62 \mu \mathrm{M})$ and a $K_{\mathrm{sv}}$ value of as high as $4 \times 10^{4} \mathrm{M}^{-1}$ was calculated.

Very recently, Xie and co-workers reported two highly stable and strongly fluorescent MOFs, $\left[\mathrm{Zr}_{6} \mathrm{O}_{4}(\mathrm{OH})_{8}\left(\mathrm{H}_{2} \mathrm{O}\right)_{4}\left(\mathrm{~L}^{1}\right)_{2}\right]$ (BUT-14) and $\left[\mathrm{Zr}_{6} \mathrm{O}_{4}(\mathrm{OH})_{8}\left(\mathrm{H}_{2} \mathrm{O}\right)_{4}\left(\mathrm{~L}^{2}\right)_{2}\right]$ (BUT-15), crafted from two new organic linkers (Fig. 10a). ${ }^{100}$ These isostructural robust MOFs displayed exclusive luminescence quenching for $\mathrm{Fe}^{3+}$ ions in water. Both MOFs were found to show excellent selectivity with interfering ions and good recyclability (6 cycles) towards $\mathrm{Fe}^{3+}$ sensing (Fig. 10b). Additionally, a very low limit of detection (212 ppb for BUT-14 and $16 \mathrm{ppb}$ for BUT-15) was calculated for both MOFs, and BUT-15 showed unaltered efficiency for $\mathrm{Fe}^{3+}$ sensing in simulated biological conditions. Another new carbazole functionalized $\mathrm{Zr}$ (Iv) MOF-based $\mathrm{Fe}^{3+}$ sensor, $\left[\mathrm{Zr}_{6} \mathrm{O}_{4}(\mathrm{OH})_{4}(2,7-\mathrm{CDC})_{6}\right] \cdot 19 \mathrm{H}_{2} \mathrm{O} \cdot 2 \mathrm{DMF}$ (1), with the UiO-67 topology was reported by Biswas and co-workers. ${ }^{101}$ The probe displayed efficient $\mathrm{Fe}^{3+}$ sensing with a $K_{\mathrm{sv}}$ value of $5.5 \times 10^{4} \mathrm{M}^{-1}$ and good reusability towards $\mathrm{Fe}^{3+}$ sensing. The same group further extended their study by reporting a new $\mathrm{Hf}$ (Iv)-based MOF possessing a UiO-66 topology, which could selectively recognize $\mathrm{Fe}^{3+}$ ions in water using 3-methyl-4-phenylthieno[2,3-b]thiophene-2,5-dicarboxylic acid ( $\left.\mathrm{H}_{2} \mathrm{MPTDC}\right)$ as the organic linker and a $\left[\mathrm{Hf}_{6} \mathrm{O}_{4}(\mathrm{OH})_{4}\right]^{12+}$ SBU. ${ }^{102}$ A good quenching efficiency $\left(K_{\mathrm{sv}}=8.22 \times 10^{3} \mathrm{M}^{-1}\right)$ together with the detection limit of $2.7 \times 10^{-7} \mathrm{M}$ was calculated for $\mathrm{Fe}^{3+}$.

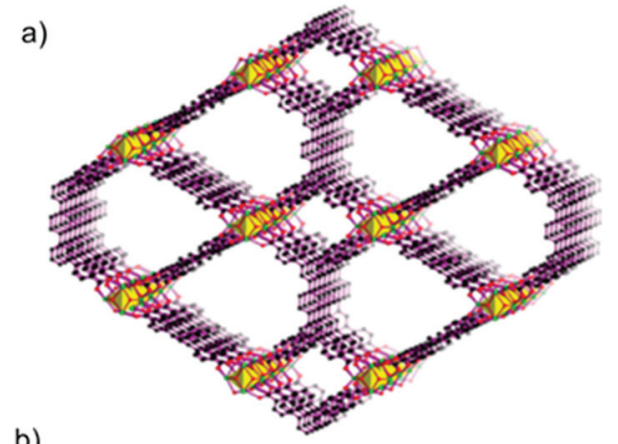

b)

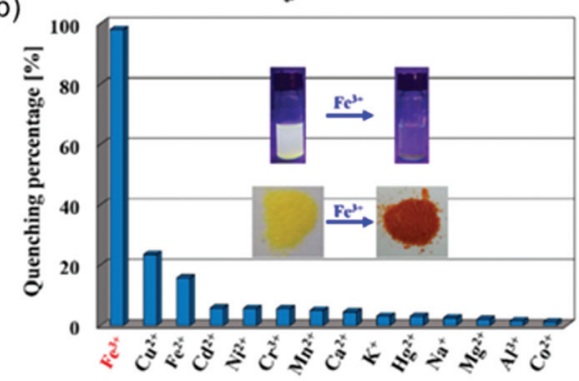

Fig. 10 (a) Framework structure of BUT-14 showing quadrangular and pear-like channels. (b) Plot showing \% fluorescence quenching in BUT-15 with $1 \mathrm{mM}$ various metal ions at $320 \mathrm{~nm}$ excitation in water medium (inset, images of BUT-15 and BUT-15- $\mathrm{Fe}^{3+}$ in water under $365 \mathrm{~nm}$ UV light, and images of them as a solid). Reproduced with permission from ref. 100. Copyright 2017, American Chemical Society.
In another report Cheng, Yang and co-workers introduced a novel and convenient $\mathrm{Fe}^{3+}$ colorimetric sensor in aqueous medium by utilizing ultra-small zero-dimensional Zn-MOF-74 [ $\mathrm{Zn}_{2}$ (DOBDC), DOBDC: 2,5-dihydroxyterephthalic acid] nanodots. ${ }^{103}$ The prepared nanodots combined with their very high dispersity in water and great surface-to-volume ratio illustrated enhanced sensitivity towards the detection of $\mathrm{Fe}^{3+}$ originating from their close interaction with the target analyte. A very low LOD $(1.04 \mu \mathrm{M})$ was calculated for the Zn-MOF-74 nanodots and the sensing could be easily monitored via naked eye colour changes. Additionally, the sensing mechanism based on framework collapse and blue Fe-DOBDC salt complex formation was also demonstrated. To overcome the impediment associated with processability in MOFs Du, Fang and coworkers came up with a unique strategy of loading an aminofunctionalized mesoporous MOF (453-MOF) in a polymer matrix following an in situ co-polymerization technique. ${ }^{104}$ The prepared MOF-polymer composite was easily processable and behaved as a fluorescent detector for $\mathrm{Fe}^{3+}$ ions with improved selectivity compared to the pristine MOF.

Adding to the sensing literature, our group recently reported a MOF@Dye composite aimed at $\mathrm{Fe}^{3+}$ sensing in water medium. ${ }^{105}$ A water stable anionic MOF, viz. Bio-MOF-1 $\left[\mathrm{Zn}_{8}(\mathrm{ad})_{4}(\mathrm{BPDC})_{6} \mathrm{O} \cdot 2 \mathrm{Me}_{2} \mathrm{NH}_{2}\right] \cdot \mathrm{G}$ (G: DMF and water, BPDC: biphenyl dicarboxylic acid), was chosen as a host to strategically encapsulate the fluorescent cationic dye Rhodamine B (RhB) with accessible free carboxylic acid for strongly interacting with the target analyte (Fig. 11a). The MOF@Dye composite was tested for $\mathrm{Fe}^{3+}$ sensing in water and exhibited remarkable selectivity $\left(K_{\mathrm{sv}}: 5.5 \times 10^{4} \mathrm{M}^{-1}\right)$ with several high concentration interfering cations as well as high sensitivity (LOD =

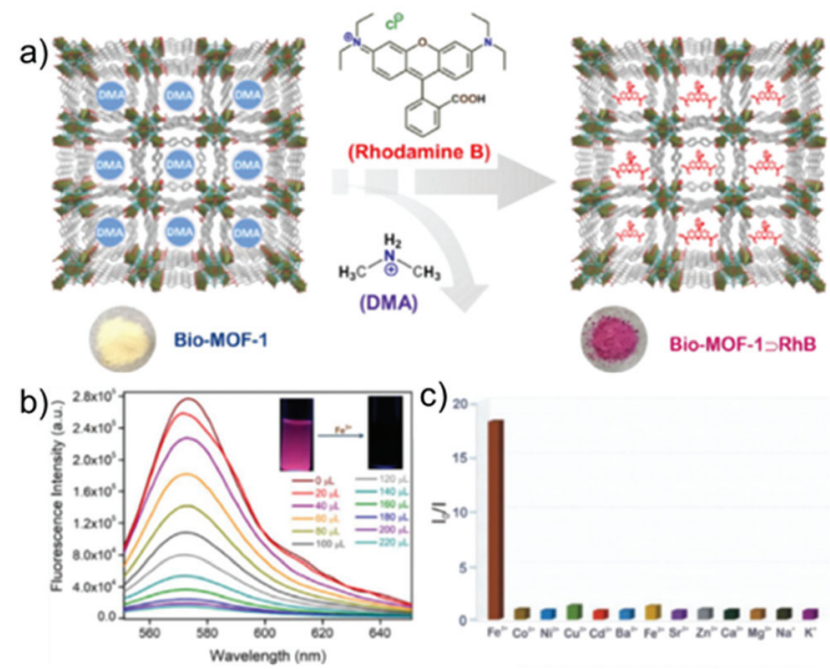

Fig. 11 (a) Representative schematic view of encapsulating Rhodamine $\mathrm{B}(\mathrm{RhB})$ dye inside Bio-MOF-1. (b) Photoluminescence intensity change in Bio-MOF-1@RhB upon the gradual addition of Fe(III) ions in water under excitation at $540 \mathrm{~nm}$. (c) Extent of fluorescent quenching response of Bio-MOF-1aRhB in the presence of different metal ions in water at $540 \mathrm{~nm}$ excitation. Reproduced with permission from ref. 105. Copyright 2020 Elsevier. 
$1.1 \mathrm{ppm}$ ) (Fig. 11b). Furthermore, the fluorescent probe was found to be reusable towards $\mathrm{Fe}^{3+}$ sensing for up to 4 cycles. Following this work, our group also reported another efficient $\mathrm{Fe}^{3+}$ sensor via the utilization of the basic pyridyl functionality in UiO-66@N MOF built from 2,5-pyridine dicarboxylic acid. ${ }^{106}$ The N-containing MOF exhibited excellent selective $\mathrm{Fe}^{3+}$ sensing $\left(K_{\mathrm{sv}}: 6.65 \times 10^{3} \mathrm{M}^{-1}\right)$ and appreciably low detection limit $(\mathrm{LOD}=0.69 \mathrm{ppm})$ together with reusability. Also, there are various other reports in the literature dedicated towards $\mathrm{Fe}^{3+}$ sensing using MOFs. ${ }^{107-110}$

Moreover, Yan and co-workers disclosed an otherwise difficult sensing of ferrous $\left(\mathrm{Fe}^{2+}\right)$ ions over ferric ions $\left(\mathrm{Fe}^{3+}\right)$ by employing a previously reported fluorescent MOF, viz. nMOF-253s (Al(OH)(bpydc) 2.6DMF, bpydc: 2,2'-bipyridine-5,5'dicarboxylic acid). ${ }^{111}$ Three types of nanoscale MOFs (nMOF-253s) were synthesized with varying particle sizes (300 to $50 \mathrm{~nm})$, and it was found that MOF-253 ( $\gamma)$ with a particle size of exhibited a luminescence quenching response in water exclusively for $\mathrm{Fe}^{2+}$ ions. The authors also demonstrated intracellular $\mathrm{Fe}^{2+}$ detection by incorporating MOF-253 $(\gamma)$ inside HeLa cells and suggested that a PET-based mechanism may be responsible for the selective $\mathrm{Fe}^{2+}$ sensing over $\mathrm{Fe}^{3+}$ and other metal ions.

3.1.2b Copper ( $\mathrm{Cu}$ ) sensing. Besides iron (Fe), copper $(\mathrm{Cu})$ is also a fundamental metal in biological applications owing to its crucial enzymatic behavior in living organisms at a low concentration. Besides, the emergence of discrepancy in copper concentration especially in excess can cause explicit neurodegenerative disorders such as Parkinson's disease and Wilson's disease, thus necessitating the need for the development of potent optical sensors. ${ }^{112,113} \mathrm{Chi}$ and co-workers developed a new strongly fluorescent MOF-based $\mathrm{Cu}^{2+}$ sensor via an encapsulation strategy and fabricated a composite material by embedding branched poly-(ethylenimine)-capped carbon quantum dots (BPEI-CQDs) inside the porous ZIF-8 scaffold. ${ }^{114}$ The as-synthesized blue fluorescent composite was then probed for $\mathrm{Cu}^{2+}$ sensing via luminescence quenching and was found to illustrate good potency with a broad range response (2-1000 nM) together with a very low LOD (80 pM). Further, the BPEI-CQDS/ZIF-8 composite demonstrated satisfactory $\mathrm{Cu}^{2+}$ detection in real water samples accompanied by appreciable selectivity amongst competing ions. Furthermore, ionexchange based $\mathrm{Cu}^{2+}$ sensing via fluorescence quenching was reported by Maji and co-workers in a new anionic MOF $\left\{\left[\mathrm{Mg}_{3}(\mathrm{ndc})_{2.5}\left(\mathrm{HCO}_{2}\right)_{2}\left(\mathrm{H}_{2} \mathrm{O}\right)\right]\left[\mathrm{NH}_{2} \mathrm{Me}_{2}\right] \cdot 2 \mathrm{H}_{2} \mathrm{O} \cdot \mathrm{DMF}\right\}$ (ndc: 1,4naphthalenedicarboxylate). ${ }^{115}$ This compound exhibited a significant "turn-off" response toward $\mathrm{Cu}^{2+}$ ions $(1.986 \times$ $103 \mathrm{M}^{-1}$ ) and the static quenching was explained based on the complexation of $\mathrm{Cu}^{2+}$ with the carboxylate oxygens accompanied by structural reorganization.

Recently Jiang and co-workers developed a unique strategy to detect $\mathrm{Cu}^{2+}$ ions with a more preferable "turn-on" response in a strategically chosen Zr-MOF, viz. PCN-222, crafted from $\mathrm{Zr}^{4+}$ metal nodes connected with a tetrakis(4-carboxyphenyl) porphyrin ( $\left.\mathrm{H}_{2} \mathrm{TCPP}\right)$ organic linker. ${ }^{116}$ The free base porphyrin was then utilized to immobilize $\mathrm{Pd}^{2+}$ cations to yield PCN-222-
Pd(II), which illustrated selective fluorescence enhancement via a "turn-on" response in aqueous medium (Fig. 12a). The observed enhancement was credited to the strong affinity of $\mathrm{Cu}^{2+}$ ions toward the porphyrin $\mathrm{N}$-atoms, leading to the discharge of an equivalent amount of palladium nanoparticles stabilized by the framework scaffold (Fig. 12b). The as-formed Pd-NPs then act as a potent catalyst for the Heck reaction in transforming non-fluorescent-substituted aniline substrates to fluorescent indole molecules to serve as a read-out signal. An acceptable limit of detection (50 nm) was obtained, which was lower than the limit set by the Environmental Protection Agency (EPA) in drinking water. Liu and co-workers explored another porphyrin-based MOF, namely MOF-525 $\mathrm{Zr}_{6} \mathrm{O}_{4}(\mathrm{OH})_{4}\left(\mathrm{TCPP}-\mathrm{H}_{2}\right)_{3}$ (TCPP: meso-tetra(4-carboxyphenyl)porphyrin), as a fast responsive fluorescence probe for $\mathrm{Cu}^{2+}$ ions. ${ }^{117}$ The porphyrin moiety decorated with four $\mathrm{N}$ atoms presented an excellent recognition site for a selective and sensitive response toward $\mathrm{Cu}^{2+}$ ions. MOF-525 exhibited a very small "turn-off" response time of $40 \mathrm{~s}$ together with a high $K_{\mathrm{sv}}$ value of $4.5 \times 10^{5} \mathrm{M}^{-1}$ and low LOD of $67 \mathrm{nM}$. Furthermore, the fluorescence quenching was constant for only $\mathrm{Cu}^{2+}$ and not for other interfering metal cations. The selectivity was attributed to the higher affinity of the porphyrin core toward $\mathrm{Cu}^{2+}$ compared to other metal ions, while a dynamic mechanism was suggested to be operational via $\mathrm{Cu}(\mathrm{II})$-porphyrin complex formation, as supported by lifetime measurements.

Chen and co-workers demonstrated for the first time, naked-eye $\mathrm{Cu}^{2+}$ detection in a SC-SC manner in a Cd-MOF $\left\{\mathrm{NH}_{2}\left(\mathrm{CH}_{3}\right)_{2} \cdot \mathrm{Cd}_{2.5}(\mathrm{~L})_{2}\left(\mathrm{H}_{2} \mathrm{O}\right) \cdot\left(\mathrm{H}_{2} \mathrm{O}\right)\right\}_{n}\left(1 ; \mathrm{H}_{3} \mathrm{~L}\right.$ : tricarboxytriphenyl-
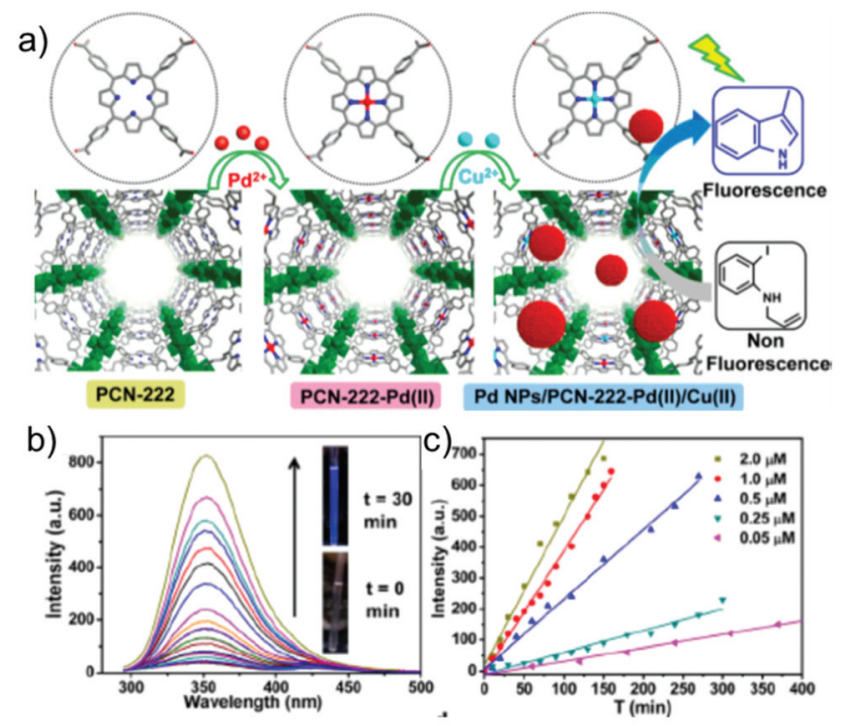

Fig. 12 (a) Schematic representation of the preparation of PCN-222-Pd (II) and finally PD NPs/PCN-222-Pd(II)/Cu(II). (b) Luminescence enhancement of the probe upon addition of $\mathrm{Cu}^{2+}$ ions (inset: images of the solution before and after the catalytic reaction under UV light). (c) Linear relationship of fluorescence intensity enhancement as a function of time at different concentrations of $\mathrm{Cu}^{2+}$ ions. Reproduced with permission from ref. 116. Copyright, 2016 American Chemical Society. 
amine). An instant yellow to dark green colour change was observed with a remarkable response time of $9 \mathrm{~s}$ for 1 with a $\mathrm{Cu}^{2+}$ concentration of $10^{-4} \mathrm{~mol} \mathrm{~L}{ }^{-1}$, as validated by in situ microcalorimetry. ${ }^{118}$ Additionally, this response was exclusive for $\mathrm{Cu}^{2+}$ ions since other metal cations yielded no significant colour change, and amorphization was also observed. It is also noteworthy that this MOF-based chemosensor provides an advantage over other probes in response time and usability in water medium. Recently, Wang and co-workers fabricated a highly fluorescent carbon dot@MOF composite for the specific recognition of $\mathrm{Cu}^{2+}$ by ratiometric fluorescent sensing. ${ }^{119}$ The synthesized ball-flower-like nanostructures of CDs@Eu-DPA (DPA: 2,6-pyridinedicarboxylic acid) displayed two characteristic emissions at $615 \mathrm{~nm}$ and $425 \mathrm{~nm}$ when excited at $275 \mathrm{~nm}$. Upon the addition of an aqueous solution of $\mathrm{Cu}^{2+}$, the peak maximum at $615 \mathrm{~nm}$ showed a gradual decrease, while the peak at $425 \mathrm{~nm}$ remained unaltered, thus leading to a ratiometric fluorescence response. Also, the selectivity was further validated with insignificant interference on F425/F415 by competing metal ions together with a very low LOD of $26.3 \mathrm{nM}$. Besides the aforementioned reports, a few more articles have been reported on the detection of $\mathrm{Cu}^{2+}$ with MOFs. ${ }^{120-123}$

3.1.2c Aluminium (Al) sensing. It is well known that aluminium is one of the most abundant metal elements in the Earth's crust. ${ }^{124}$ Aluminium metal contamination has also attracted much attention because of its toxicity to living systems. Although a trace amount of aluminium is required in the human body, when it accumulates in high quantity it can cause severe health diseases. Among the diseases caused by aluminium toxicity, Alzheimer's disease and Parkinson's disease are well known. ${ }^{124}$ Thus, these adverse effects of aluminium has led to the search for sensors that can detect $\mathrm{Al}^{3+}$ ions selectively and rapidly. Sun and co-workers introduced a luminescent anionic Ln-MOF, $\left[\mathrm{H}_{2} \mathrm{~N}\left(\mathrm{CH}_{3}\right)_{2}\right]$ $\left[\mathrm{Eu}\left(\mathrm{H}_{2} \mathrm{O}\right)_{2}(\mathrm{BTMIPA})\right] \cdot 2 \mathrm{H}_{2} \mathrm{O}$ (1) ( $\mathrm{H}_{4}$ BTMIPA: 5, 5'-methylenebis (2,4,6-trimethylisophthalic acid)), for the selective detection of $\mathrm{Al}^{3+}$ and $\mathrm{Fe}^{3+} \cdot{ }^{124}$ The tubular channels housing the $\left[\mathrm{H}_{2} \mathrm{~N}\right.$ $\left.\left(\mathrm{CH}_{3}\right)_{2}\right]^{+}$ions presented an opportunity to study the ionexchange-based luminescence behaviour. The authors noted that most of the tested metal ions had different extents of quenching response (highest for $\mathrm{Fe}^{3+}$ ), while there was a luminescence enhancement in the case of $\mathrm{Al}^{3+}$ ions in DMF solution. Further, test plates were constructed to make the sensing process more convenient, and the mechanism of fluorescence enhancement for $\mathrm{Al}^{3+}$ was also discussed.

Extending the potential of Ln-MOFs toward cation sensing, Zang and co-workers synthesized a hydrolytically stable honeycomb-type MOF, viz. $\left\{\left[\mathrm{Tb}(\mathrm{L} 1)_{1.5}\left(\mathrm{H}_{2} \mathrm{O}\right)\right] \cdot 3 \mathrm{H}_{2} \mathrm{O}\right\}_{n}$ (Tb-MOF) (2-(2hydroxy-propionylamino)-terephthalic acid), for the detection of $\mathrm{Al}^{3+}$ ions in aqueous solution. ${ }^{125}$ The sensing of $\mathrm{Al}^{3+}$ ions involved a unique fluorescence enhancement in the ligand. An intensification in luminescence was observed only for $\mathrm{Al}^{3+}$ ions, while the mechanism was suggested to be attributed to framework collapse.

Recently $\mathrm{Bu}, \mathrm{Hu}$ and co-workers reported a "turn-on" $\mathrm{Al}^{3+}$ sensor based on a Zn-MOF, $\left\{\left[\mathrm{Zn}_{2}(\mathrm{O}-\mathrm{BTC})\left(4,4^{\prime}-\right.\right.\right.$
BPY $\left.\left.)_{0.5}\left(\mathrm{H}_{2} \mathrm{O}\right)_{3}\right] \cdot\left(\mathrm{H}_{2} \mathrm{O}\right)_{1.5} \cdot(\mathrm{DMA})_{0.5}\right\}_{n}$ (NUM-2) (O-BTC: 2-hydroxybenzene-1,3,5-tricarboxylic acid, BPY: 4,4'-bipyridine), crafted on a mixed-ligand strategy. ${ }^{126}$ NUM-2 exhibited enhanced luminescence exclusively for $\mathrm{Al}^{3+}$ ions in ethanol medium even in the presence of competing analytes with good sensitivity $(\mathrm{LOD}=0.10 \mathrm{ppm})$. Additionally, the plausible mechanism was also proposed.

Earlier this year, Morsali and co-workers came up with an inventive strategy for the detection and quantification of $\mathrm{Al}^{3+}$ ions both in aqueous and non-aqueous media. ${ }^{127}$ The reported unique sacrificial MOF, (TMU-60: $\left.\mathrm{Zn}(\mathrm{OBA})\left(\mathrm{L}^{*}\right)\right] \cdot \mathrm{DMF}\left(\mathrm{L}^{*}:\right.$ 5,6dipyridin-4-yl-1,2,3,4-tetrahydropyrazine, OBA: 4,4'-oxybisbenzoic acid) displayed unique interactions with $\mathrm{Al}^{3+}$, which trigger framework degradation in a quantitative manner followed by a naked-eye detectable swift color change (colorless to red) in the solvent. Further, this unique response was observed only for $\mathrm{Al}^{3+}$, even in presence of other metal ions, with a fast response time ( $<3 \mathrm{~min})$ and also minute $\mathrm{Al}^{3+}$ concentration ( $>500 \mathrm{ppb}$ ). Additionally, this convenient naked-eyeenabled sensor had a good LOD of $100 \mathrm{ppb}$, while the mechanism of the "turn-on" response for $\mathrm{Al}^{3+}$ was also discussed.

3.1.2e Uranyl $\left(\mathrm{UO}_{2}{ }^{2+}\right)$ sensing. Th sensing of radioactive uranium $\left({ }^{235} \mathrm{U}\right.$ and $\left.{ }^{238} \mathrm{U}\right)$ has gained paramount interest in

a)

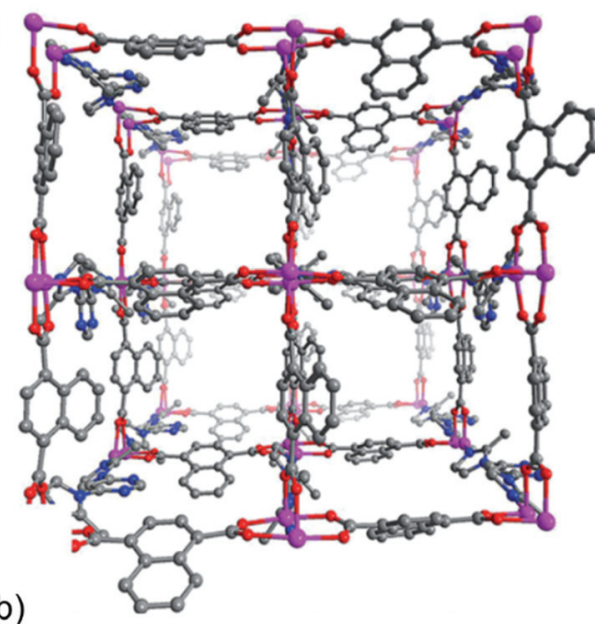

b)

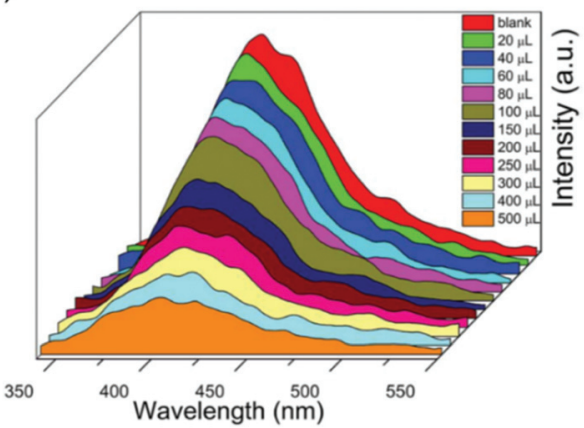

Fig. 13 (a) Ball-and-stick representation of the single structure of MOF. (b) Emission profile of the MOF dispersed in a DMF : water solution ( $\mathrm{v} / \mathrm{v}$ 25 : 1) upon the incremental addition of $\mathrm{UO}_{2}{ }^{2+}$ ions $(1 \mathrm{mM})$. Reproduced with permission from ref. 128. Copyright 2017 Royal Society of Chemistry. 
recent years because of its toxicity towards living systems together with its high half-life (half-life for ${ }^{235} \mathrm{U}$ and ${ }^{238} \mathrm{U}$ are 7 $\times 10^{8}$ years and $4.5 \times 10^{9}$ years, respectively). Sun and coworkers reported a 3D MOF-bearing pcu network namely $\left[\mathrm{Co}_{2}(\mathrm{dmimpym})(\mathrm{nda})_{2}\right]_{n}$ (1; dmimpym: 4,6-di(2-methyl-imidazol-1-yl)-pyrimidine, $\mathrm{H}_{2}$ nda: 1,4-naphthalenedicarboxylic acid) with an accessible basic pyrimidyl moiety for recognizing environmentally hazardous $\mathrm{UO}_{2}{ }^{2+}$ in DMF : water $(\mathrm{v}: \mathrm{v}=$ $25: 1) .{ }^{128}$ An efficient "turn-off" response was observed with a high $K_{\mathrm{sv}}$ value $\left(1.1 \times 10^{4} \mathrm{M}^{-1}\right)$, while the interaction between $\mathrm{UO}_{2}{ }^{2+}$ and the pyrimidyl sites was mentioned as the probable reason for the luminescence quenching (Fig. 13).

3.1.2d Silver $(\mathrm{Ag})$ sensing. Apart from the aforementioned sensing applications of MOFs and MOF-based materials, $\mathrm{Ag}^{+}$ ions sensing with MOFs has also been reported recently. Yan and co-workers adopted a PSM strategy to fabricate new types of Ln-MOFs ( $\mathrm{Ln}=\mathrm{Sm}$, Dy, Nd, Yb, and Er) through the encapsulation of $\mathrm{Ln}^{3+}$ cations in a reported MOF, MIL-121, $(\mathrm{Al}(\mathrm{OH})$ $\left(\mathrm{H}_{2}\right.$ btec $) \cdot \mathrm{H}_{2} \mathrm{O}, \mathrm{H}_{4}$ btec: pyromellitic acid) to function as a potential $\mathrm{Ag}^{+}$sensor. $^{129}$ The authors found that $\mathrm{Sm}^{3+}$ @MIL-121 could detect $\mathrm{Ag}^{+}$via fluorescence enhancement, while other metal ions barely showed any noticeable fluorescence

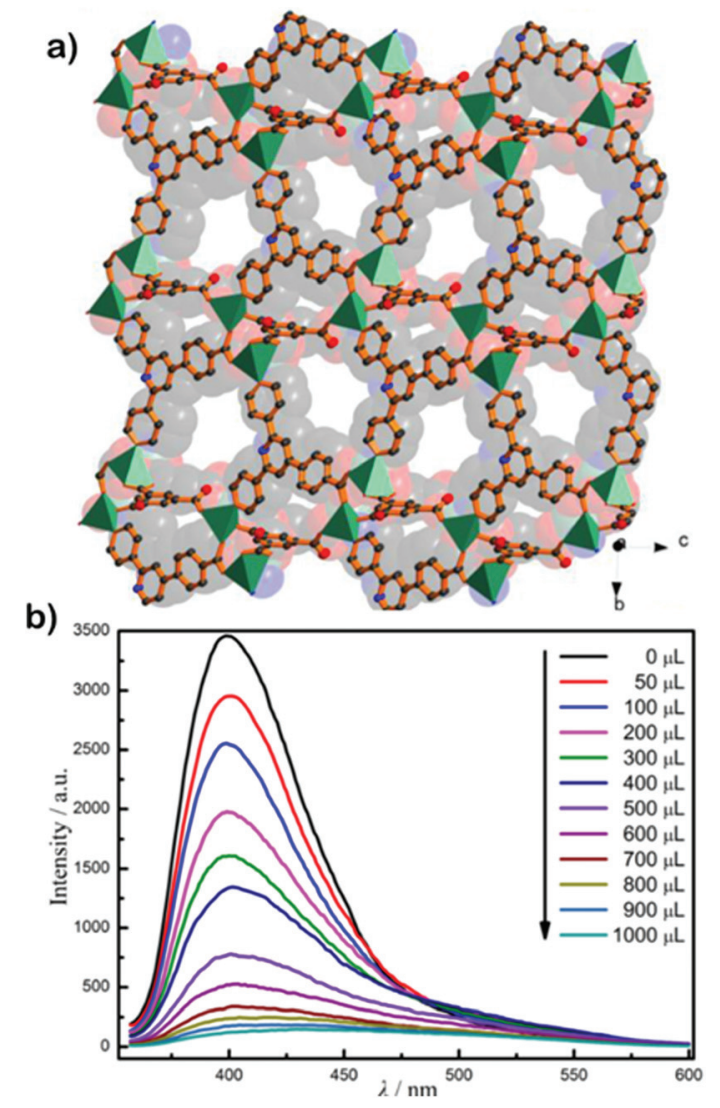

Fig. 14 (a) 3D framework of the MOF along the a-axis. (b) Concentration-dependent emission spectra of the probe with paraquat in aqueous solution. Reproduced with permission from ref. 109. Copyright 2019 John Wiley \& Sons. response, demonstrating the excellent selectivity of the probe. The LOD of Sm${ }^{3+} @$ MIL-121 was found to be $0.09 \mu \mathrm{M}$ for $\mathrm{Ag}^{+}$.

\subsection{Recognition of organic cationic pollutants}

Apart from the aforementioned inorganic cationic pollutants, some organic cationic water pollutants are also known as very toxic and hazardous species. Especially, organic herbicides such as paraquat (PQ) and diquat (DQ) have been found to be very toxic in nature and can cause several diseases. Being ionic in nature, both PQ and DQ are known to be highly soluble in water. Paraquat was synthesized for the first time in 1882, and it is recognized as the most commonly used herbicide to date. Owing to their high solubility in water, these compounds can pollute different water bodies through the release of sewages from various farms. In addition, the half-life of paraquat can be up to 20 years. Furthermore, paraquat toxicity has been observed to cause Parkinson's disease and can also lead to death. ${ }^{130}$ Consequently, the detection of these toxic cationic organic pollutants is very essential.

In a recent report, Chen et al. reported the detection of paraquat with a luminescent $\mathrm{Zn-MOF}$, namely, [ $\mathrm{Zn}_{2}$ (cptpy)(btc) $\left.\left(\mathrm{H}_{2} \mathrm{O}\right)\right]_{n}{ }^{109}$ This MOF was synthesized through a mixed-linker strategy with $\mathrm{Zn}$ (II) ions, where, Hcptpy and $\mathrm{H}_{3}$ btc were used as the organic ligands (Hcptpy: 4-(4-carboxyphenyl)-2,2':4',4"-terpyridine and $\mathrm{H}_{3}$ btc: 1,3,5-benzenetricarboxylic acid). This
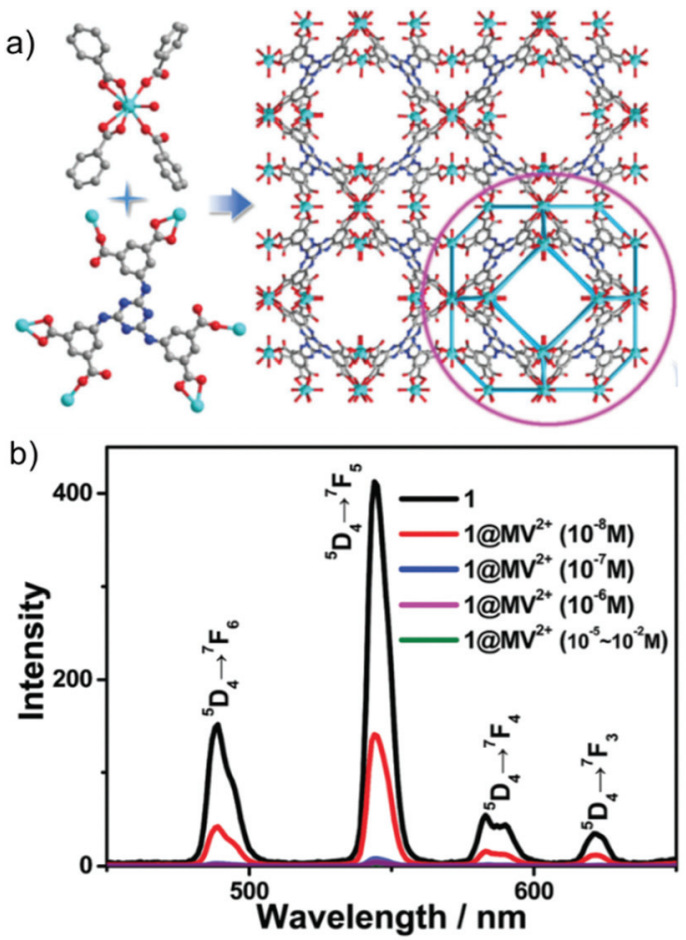

Fig. 15 (a) Coordination environment of the $\mathrm{Tb}^{3+}$ ions and the coordination model of the TATAT $^{6-}$ ligands in the three-dimensional structure of the MOF. (b) Solid-state emission spectra of the MOF and soaked samples of the MOF in $\mathrm{MV}^{2+}$ aqueous solutions with different concentrations. Reproduced with permission from ref. 131. Copyright 2018 Royal Society of Chemistry. 
MOF was found to be three-dimensional with one-dimensional channels inside it (Fig. 14a). Furthermore, this MOF was observed to be stable in water and in the $\mathrm{pH}$ range of $\mathrm{pH}-3$ to
$\mathrm{pH}-11$. An emission band at $400 \mathrm{~nm}$ was obtained from the MOF upon excitation at $340 \mathrm{~nm}$, which was speculated to be generated from the intra-ligand emission state of $\pi^{*} \rightarrow \mathrm{n}$ and/

Table 1 List of some MOFs showing a response in the presence of cationic species

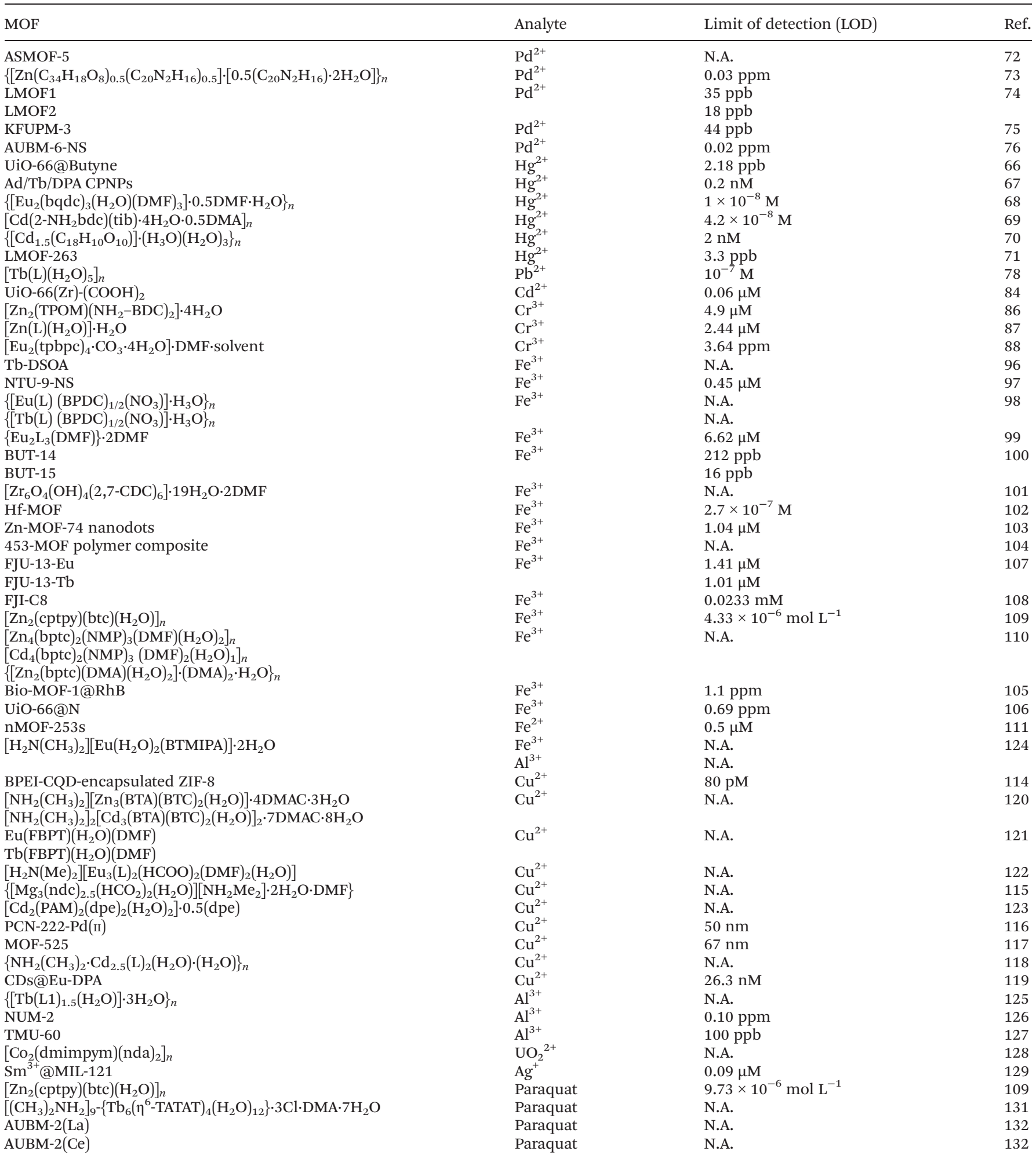

N.A. $=$ information not available. 
or $\pi^{*} \rightarrow \pi$ transition. This MOF showed sensitivity towards $\mathrm{Fe}^{3+}$ ions in water medium. Further, the detection of paraquat with the MOF was carried out in water medium also. The photoluminescence profile of the MOF was found to be quenched upon the incremental addition of paraquat, and with $1.296 \mathrm{mmol} \mathrm{L}^{-1}$ of the analyte, almost $96.2 \%$ quenching was obtained (Fig. 14b). The Stern-Volmer constant $\left(K_{\text {sv }}\right)$ was calculated to check the quenching efficiency of the probe, which was found to be $1.038 \times 103 \mathrm{~L} \mathrm{~mol}^{-1}$. In addition, the detection limit of paraquat with the sensory material was calculated to be $9.73 \times 10-6 \mathrm{~mol} \mathrm{~L}^{-1}$. The reusability of the probe was confirmed by the recycle experiment, where the MOF showed comparable quenching efficiency in the presence of paraquat even up to three cycles.

In another report, Du et al. demonstrated Tb-MOF, with a molecular formula of $\left[\left(\mathrm{CH}_{3}\right)_{2} \mathrm{NH}_{2}\right]_{9}-\left\{\mathrm{Tb}_{6}\left(\eta^{6} \text {-TATAT }\right)_{4}\left(\mathrm{H}_{2} \mathrm{O}\right)_{12}\right\}$. 3Cl-DMA $7 \mathrm{H}_{2} \mathrm{O}$ (where, $\mathrm{H}_{6}$ TATAT: 5,5',5"-(1,3,5-triazine-2,4,6triyltriimino)tri-1,3-benzenedicarboxylic acid), for the detection of paraquat. The MOF was found to consist of an anionic framework, where the residual charge was balanced by a dimethyl ammonium cation $\left[\left(\mathrm{CH}_{3}\right)_{2} \mathrm{NH}_{2}{ }^{+}\right]$inside (Fig. 15a). ${ }^{131}$ This Tb-MOF consisted of one large closed hexagonal window with dimensions of $\sim 19.4 \times 19.4 \AA^{2}$ and two small square open windows with dimensions of $\sim 11.6 \times 11.6 \AA^{2}$ and $\sim 7.8 \times 7.8 \AA^{2}$, respectively. In addition, a large cavity of $31.0 \times 31.0 \times 31.0 \AA^{3}$ was observed to be present inside the Tb-MOF. The MOF was found to produce nice green emission under $365 \mathrm{~nm}$ UV-light. The emission peaks of the compound were observed at 489 , 544, 583 and $621 \mathrm{~nm}$ upon excitation at $346 \mathrm{~nm}$ in the solid state. These respective emission peaks were corroborated with the ${ }^{5} \mathrm{D}_{4} \rightarrow{ }^{7} \mathrm{~F}_{J}$ (where, $J=6-3$ ) transitions and the origin of the luminescence was speculated to be from the $\mathrm{Tb}^{3+}$ ion. Owing to the presence of the extra framework cation (dimethyl ammonium cation) inside the network, paraquat was incorporated via cation exchange, where colour change was obtained. Thereafter, solid-state luminescence was measured with the paraquat-incorporated phase of the Tb-MOF, where quenching of the luminescence was observed. Then for detailed studies, Tb-MOF was soaked in different concentrations of paraquat solution $\left(10^{-2}, 10^{-3}, 10^{-4}, 10^{-5}, 10^{-6}, 10^{-7}\right.$, and $\left.10^{-8} \mathrm{M}\right)$ for $10 \mathrm{~min}$. The rapid quenching was monitored via solid-state fluorescence spectroscopy upon the incorporation of the paraquat inside the framework. A noticeable quenching was observed even with $10^{-8} \mathrm{M}$ concentration of paraquat, whereas, the luminescence of Tb-MOF was found to be quenched almost completely with $10^{-7} \mathrm{M}$ of the analyte (Fig. 15b).

In addition, Hmadeh and co-workers reported another type of lanthanide-based MOF for the detection of metal ions and paraquat recently. ${ }^{132}$ In their report, two sets of MOFs were synthesized with two lanthanide cations $\left(\mathrm{La}^{3+}\right.$ and $\left.\mathrm{Ce}^{3+}\right)$ together with two organic linkers, namely NP (2,6-naphthalenedicarboxylic acid) and DNP (1,5-dihydroxy-2,6-naphthalenedicarboxylic acid). As a combination of these two, a total of four MOFs were synthesized in this work, viz., AUBM-2 (Ce) and AUBM-2(La) for NP linker and AUBM-3(Ce) and AUBM-3
(La) for DNP linker. After thorough characterisation, the four MOFs were employed for the sensing of heavy metal ions $\left(\mathrm{Hg}^{2+}, \mathrm{Cd}^{2+}, \mathrm{Pb}^{2+}, \mathrm{Cr}^{3+}, \mathrm{As}^{3+}\right.$, etc. $)$. Furthermore, these compounds were used for the sensing of paraquat in the solution phase. Upon the addition of paraquat, the fluorescence emission profiles for both AUBM-2(La) and AUBM-2(Ce) were completely quenched. Especially, quenching was found to be very rapid for AUBM-2(La) compared to that of AUBM-2(Ce). In addition, the quenching constants from the Stern-Volmer plot $\left(K_{\mathrm{sv}}\right)$ for AUBM-2(La) and AUBM-2(Ce) were calculated to be 21 $\mathbf{M}^{-1}$ and $9 \mathbf{M}^{-1}$, respectively. On the other hand, AUBM-3(La) was also found to exhibit similar properties to AUBM-2(La) with s similar $K_{\mathrm{sv}}$ value of $\sim 21 \mathrm{M}^{-1}$. It was speculated that the charge transfer between the electron rich linkers of the MOFs and electron-deficient paraquat result in the quenching phenomenon. Furthermore, it was observed that at a low concentration, dynamic quenching behaviour was exhibited, while at a higher concentration of the analyte, both dynamic and static quenching behaviour was observed.

Furthermore, in an earlier report, $\mathrm{Bu}$ and co-workers demonstrated the capture of paraquat (PQ) and diquat (DQ) with an anionic framework-based Zn-MOF, namely, NKU-101, with the molecular formula of $\left[\left(\mathrm{CH}_{3} \mathrm{CH}_{2}\right)_{2} \mathrm{NH}_{2}\right]_{1 / 2}[\mathrm{Zn}$ $\left.(\mathrm{BTC})_{2 / 3}(\mathrm{PyC})_{1 / 4}\right] \cdot$ solvent (where, NKU: Nankai University; $\mathrm{H}_{2}$ PyC: 4-pyrazolecarboxylic acid and $\mathrm{H}_{3}$ BTC: 1,3,5-benzenetricarboxylic acid). ${ }^{133}$ Efficient removal of both PQ and DQ was obtained with NKU-101 with a capacity of 160 and $200 \mathrm{mg} \mathrm{g}^{-1}$, respectively.

\section{Conclusions and future outlooks}

In this review, we presented a brief overview summarizing the current status of metal organic frameworks (MOFs) towards cation sensing. Recent years have seen a remarkable upsurge in the augmentation of MOF-based chemosensors. Numerous luminescent metal organic frameworks (LMOFs) have been reported in the literature with remarkable performances to serve as competent sensors targeted toward several analytes such as water contaminants, heavy metal ions, biologically relevant cations and hazardous organic cations (Table 1). Moreover, some of the developed MOF-based sensors have excelled in detecting toxic and hazardous environmental pollutants with more convenient and viable approaches. Although there is comprehensive documentation of MOF-based sensory probes based on preceding reviews, here we have updated the database with recent literature reports.

Sensory probes exhibiting fluorogenic and colorimetric responses to distinct analytes provide an advantage over alternative sensors since the generated read out signal can be recognized readily using a fluorescence instrument or simply by the naked eyes. Although the research on developing extremely capable MOF-based luminescent chemosensors still remains in the nascent stage, the potential of LMOFs in analyte detection has been established strongly. In addition, LMOFs feature unique advantages, including porosity, periodic 
structure, functionality and structural versatility and tunable fluorescence. Also, several MOF-sensory probes have been constructed to detect analytes via diverse sensing mechanisms based on design rationale. The chemosensors operating on a "turn-off" response via fluorescence quenching are the most dominant for the detection of electron acceptors, where the suppression of luminescence is a consequence of the probe to analyte electron transfer, succeeded by energy transfer. On the other hand, the more preferential and convenient "turn-on" mechanism has also been realized in MOFs with guest-triggered luminescence enhancement or associated shift in wavelength, but the number of reports are very few and offers a scope for improvement. Other ways including tethering taskspecific functionality aimed at targeted analytes or integrating luminescent guests to construct multi-luminescent scaffolds featuring a dual fluorescence response not only improves the sensor quality but also helps in developing self-calibrating MOF-sensors.

Based on the reported literature, MOF-based sensors also present bottlenecks in terms of low selectivity, sensitivity, response time, reusability and stability. Consequently, the factors dictating the selectivity or sensitivity of a probe have been explored experimentally, but necessitates greater development toward real-time applicability, which include detailed assessment of probe response in real-time environments. Also, humidity/water stability and durability in biological conditions are crucial desirable features for of LMOFs, which impede the utilization of these materials under optimal real-time conditions. However, it is noteworthy to mention that striking research efforts have been dedicated to address these concerns, although more advancements are still required in developing potent sensors without performance decay over multiple cycles of sensing. Additionally, the large-scale synthesis of LMOFs with milder synthetic conditions and cheap starting materials is another area with wide scope for improvement. Hence, achieving eco-friendly synthetic protocols and exploiting cheaper precursors in crafting functionalized LMOFs with uncompromised features have attracted considerable research attention to implement practical applicability. On the other hand, LMOFs have also been able to contribute to the field by offering new avenues of research. With the augmentation of in vivo sensing and LMOF-based biomedical devices as well as the colorimetric detection of target analytes recognizable to the naked eye, LMOFs have made appreciable progress.

Although the current stats of LMOFs toward cation sensing presents plentiful innovative concepts and design principles, there is a definite window of opportunity for advancement. Several reports lack detailed studies or necessary experimental support, while various studies are not based on strategy, and rather serendipity plays a key role in the sensing of analytes. This indeed necessitates the in-depth exploration of sensoranalyte luminescence correlation aided by computational studies. Therefore, it is imperative to direct the focus of future research by developing MOF-based optical sensors in a systematic approach, taking the deign principle of the sensors as the cornerstone. Finally, the fabrication of as-synthesized
MOF-based sensors in more portable and convenient forms (e.g. thin films, device fabrication, and membrane preparation) and expanding the proof-of-concept from the lab scale to commercialized technology demand a substantial amount of research and development.

\section{Conflicts of interest}

There are no conflicts to declare.

\section{Acknowledgements}

P. S. and S. L. acknowledge UGC (India) and CSIR (India) for their fellowships respectively. W. M. and S. D. are thankful to IISER Pune for fellowships. S. K. G. acknowledges funding support from the SERB project (CRG/2019/000906).

\section{Notes and references}

1 A. J. Howarth, Y. Liu, J. T. Hupp and O. K. Farha, Metalorganic frameworks for applications in remediation of oxyanion/cation-contaminated water, CrystEngComm, 2015, 17, 7245-7253.

2 P. Samanta, A. V. Desai, S. Let and S. K. Ghosh, Advanced Porous Materials for Sensing, Capture and Detoxification of Organic Pollutants toward Water Remediation, ACS Sustainable Chem. Eng., 2019, 7, 7456-7478.

3 Drinking-water, World health organization, 2019, https:// www.who.int/news-room/fact-sheets/detail/drinking-water.

4 D. T. Sun, L. Peng, W. S. Reeder, S. M. Moosavi, D. Tiana, D. K. Britt, E. Oveisi and W. L. Queen, Rapid, Selective Heavy Metal Removal from Water by a Metal-Organic Framework/Polydopamine Composite, ACS Cent. Sci., 2018, 4, 349-356.

5 H. K. Okoro, S. O. Ayika, J. C. Ngila and A. C. Tella, Rising profile on the use of metal-organic frameworks (MOFs) for the removal of heavy metals from the environment: an overview, Appl. Water Sci., 2018, 8, 169.

$6 \mathrm{M}$. Li, H. Gou, I. Al-Ogaidi and N. Wu, Nanostructured Sensors for Detection of Heavy Metals: A Review, ACS Sustainable Chem. Eng., 2013, 1, 713-723.

7 F. Luo, J. L. Chen, L. L. Dang, W. N. Zhou, H. L. Lin, J. Q. Li, S. J. Liu and M. B. Luo, High-performance $\mathrm{Hg}^{2+}$ removal from ultra-low-concentration aqueous solution using both acylamide- and hydroxyl-functionalized metalorganic framework, J. Mater. Chem. A, 2015, 3, 9616-9620.

8 Y. Li, Y. He, F. Guo, S. Zhang, Y. Liu, W. P. Lustig, S. Bi, L. J. Williams, J. Hu and J. Li, NanoPOP: SolutionProcessable Fluorescent Porous Organic Polymer for Highly Sensitive, Selective, and Fast Naked Eye Detection of Mercury, ACS Appl. Mater. Interfaces, 2019, 11, 2739427401. 
9 Y. Wang, Y. Wu, J. Xie and X. Hu, Metal-organic framework modified carbon paste electrode for lead sensor, Sens. Actuators, B, 2013, 177, 1161-1166.

10 R. J. Drout, L. Robison, Z. Chen, T. Islamoglu and O. K. Farha, Zirconium Metal-Organic Frameworks for Organic Pollutant Adsorption, Trends Chem., 2019, 1, DOI: 10.1016/j.trechm.2019.03.010.

11 Y. Zhang, B. Yu, N. Wang and T. Li, Acute poisoning in Shenyang, China: a retrospective and descriptive study from 2012 to 2016, BMJ Open, 2018, DOI: 10.1136/ bmjopen-2018-021881.

12 D. Wu, A. C. Sedgwick, T. Gunnlaugsson, E. U. Akkaya, J. Yoon and T. D. James, Fluorescent chemosensors: the past, present and future, Chem. Soc. Rev., 2017, 46, 71057123.

13 M. C.-L. Yeung and V. W.-W. Yam, Luminescent cation sensors: from host-guest chemistry, supramolecular chemistry to reaction-based mechanisms, Chem. Soc. Rev., 2015, 44, 4192-4202.

14 H. N. Kim, W. X. Ren, J. S. Kim and J. Yoon, Fluorescent and colorimetric sensors for detection of lead, cadmium, and mercury ions, Chem. Soc. Rev., 2012, 41, 3210-3244.

15 T. Rasheed, F. Nabeel, M. Adeel, K. Rizwan, M. Bilal and H. M. N. Iqbal, Carbon nanotubes-based cues: A pathway to future sensing and detection of hazardous pollutants, J. Mol. Liq., 2019, 292, DOI: 10.1016/j.molliq.2019.111425.

16 T. Rasheed, M. Adeel, F. Nabeel, M. Bilal and H. M. N. Iqbal, $\mathrm{TiO}_{2} / \mathrm{SiO}_{2}$ decorated carbon nanostructured materials as a multifunctional platform for emerging pollutants removal, Sci. Total Environ., 2019, 688, 299-311.

17 X. Sun, Y. Wang and Y. Lei, Fluorescence based explosive detection: from mechanisms to sensory materials, Chem. Soc. Rev., 2015, 44, 8019-8061.

18 A. Karmakar, A. V. Desai and S. K. Ghosh, Ionic metalorganic frameworks (iMOFs): Design principles and applications, Coord. Chem. Rev., 2016, 307, 313-341.

19 R. E. Morris and J. Čejka, Exploiting chemically selective weakness in solids as a route to new porous materials, Nat. Chem., 2015, 7, DOI: 10.1038/NCHEM.2222.

20 A. G. Slater and A. I. Cooper, Function-led design of new porous materials, Science, 2015, 348, DOI: 10.1126/ science.aaa8075.

21 C. S. Diercks, M. J. Kalmutzki, N. J. Diercks and O. M. Yaghi, Conceptual Advances from Werner Complexes to Metal-Organic Frameworks, ACS Cent. Sci., 2018, 4, 1457-1464.

22 B. Manna, A. V. Desai and S. K. Ghosh, Neutral N-donor ligand based flexible metal-organic frameworks, Dalton Trans., 2016, 45, 4060-4072.

23 A. Schneemann, V. Bon, I. Schwedler, I. Senkovska, S. Kaskel and R. A. Fischer, Flexible metal-organic frameworks, Chem. Soc. Rev., 2014, 43, 6062-6096.

24 Z. Chang, D.-H. Yang, J. Xu, T.-L. Hu and X.-H. Bu, Flexible Metal-Organic Frameworks: Recent Advances and Potential Applications, Adv. Mater., 2015, 27, 5432-5441.
25 Y. Bai, Y. Dou, L.-H. Xie, W. Rutledge, J.-R. Li and H.-C. Zhou, Zr-based metal-organic frameworks: design, synthesis, structure, and applications, Chem. Soc. Rev., 2016, 45, 2327-2367.

26 Q.-L. Zhu and Q. Xu, Metal-organic framework composites, Chem. Soc. Rev., 2014, 43, 5468-5512.

27 B. Li, H.-M. Wen, W. Zhou, J. Q. Xu and B. Chen, Porous Metal-Organic Frameworks: Promising Materials for Methane Storage, Chem, 2016, 1, 557-580.

28 C. He, D. Liu and W. Lin, Nanomedicine Applications of Hybrid Nanomaterials Built from Metal-Ligand Coordination Bonds: Nanoscale Metal-Organic Frameworks and Nanoscale Coordination Polymers, Chem. Rev., 2015, 115(19), 11079-11108.

29 Y. Cui, J. Zhang, H. He and G. Qian, Photonic functional metal-organic frameworks, Chem. Soc. Rev., 2018, 47, 5740-5785.

30 J. Canivet, A. Fateeva, Y. Guo, B. Coasne and D. Farrusseng, Water adsorption in MOFs: fundamentals and applications, Chem. Soc. Rev., 2014, 43, 5594-5617.

31 T. Islamoglu, S. Goswami, Z. Li, A. J. Howarth, O. K. Farha and J. T. Hupp, Postsynthetic Tuning of Metal-Organic Frameworks for Targeted Applications, Acc. Chem. Res., 2017, 50, 805-813.

32 C. H. Hendon, A. J. Rieth, M. D. Korzyński and M. Dincă, Grand Challenges and Future Opportunities for MetalOrganic Frameworks, ACS Cent. Sci., 2017, 3, 554-563.

33 J. Li, X. Wang, G. Zhao, C. Chen, Z. Chai, A. Alsaedi, T. Hayat and X. Wang, Metal-organic framework-based materials: superior adsorbents for the capture of toxic and radioactive metal ions, Chem. Soc. Rev., 2018, 47, 23222356.

34 Q. Gao, J. Xu and X.-H. Bu, Recent advances about metalorganic frameworks in the removal of pollutants from wastewater, Coord. Chem. Rev., 2019, 378, 17-31.

35 K. Sumida, D. L. Rogow, J. A. Mason, T. M. McDonald, E. D. Bloch, Z. R. Herm, T.-H. Bae and J. R. Long, Carbon Dioxide Capture in Metal-Organic Frameworks, Chem. Rev., 2012, 112, 724-781.

36 T. N. Nguyen, F. M. Ebrahim and K. C. Stylianou, Photoluminescent, upconversion luminescent and nonlinear optical metal-organic frameworks: From fundamental photophysics to potential applications, Coord. Chem. Rev., 2018, 377, 259-306.

37 Y. He, F. Chen, B. Li, G. Qian, W. Zhou and B. Chen, Porous metal-organic frameworks for fuel storage, Coord. Chem. Rev., 2018, 373, 167-198.

38 S. M. Cohen, Postsynthetic Methods for the Functionalization of Metal-Organic Frameworks, Chem. Rev., 2012, 112, 970-1000.

39 A. V. Desai, S. Sharma, S. Let and S. K. Ghosh, N-donor linker based metal-organic frameworks (MOFs): Advancement and prospects as functional materials, Coord. Chem. Rev., 2019, 395, 146-192.

$40 \mathrm{~J}$. Zhou and B. Wang, Emerging crystalline porous materials as a multifunctional platform for electro- 
chemical energy storage, Chem. Soc. Rev., 2017, 46, 69276945.

41 C. Wang, X. Liu, N. K. Demir, J. P. Chen and K. Li, Applications of water stable metal-organic frameworks, Chem. Soc. Rev., 2016, 45, 5107-5134.

42 C. R. Kim, T. Uemura and S. Kitagawa, Inorganic nanoparticles in porous coordination polymers, Chem. Soc. Rev., 2016, 45, 3828-3845.

43 T. Rasheed and F. Nabeel, Luminescent metal-organic frameworks as potential sensory materials for various environmental toxic agents, Coord. Chem. Rev., 2019, 401, DOI: 10.1016/j.ccr.2019.213065.

44 M. Bilal, M. Adeel, T. Rasheed and H. M. N. Iqbal, Multifunctional metal-organic frameworks-based biocatalytic platforms: recent developments and future prospects, J. Mater. Res. Technol., 2019, 8, 2359-2371.

45 S. S. Nagarkar, A. V. Desai and S. K. Ghosh, Engineering metal-organic frameworks for aqueous phase 2,4,6-trinitrophenol (TNP) sensing, CrystEngComm, 2016, 18, 29943007.

46 P. Samanta, S. Dutta and S. K. Ghosh, Metal-Organic Frameworks (MOFs) for Environmental Applications, in Metal-organic frameworks for detection and desensitization of environmentally hazardous nitro-explosives and related high energy materials, 2019, DOI: 10.1016/B978-0-12814633-0.00002-8.

47 Y. Cui, Y. Yue, G. Qian and B. Chen, Luminescent Functional Metal-Organic Frameworks, Chem. Rev., 2012, 112, 1126-1162.

48 L. E. Kreno, K. Leong, O. K. Farha, M. Allendorf, R. P. Van Duyne and J. T. Hupp, Metal-Organic Framework Materials as Chemical Sensors, Chem. Rev., 2012, 112, 1105-1125.

49 F.-Y. Yi, D. Chen, M.-K. Wu, L. Han and H.-L. Jiang, Chemical Sensors Based on Metal-Organic Frameworks, ChemPlusChem, 2016, 81, 675-690.

50 Z. Hu, B. J. Deibert and J. Li, Luminescent metal-organic frameworks for chemical sensing and explosive detection, Chem. Soc. Rev., 2014, 43, 5815-5840.

51 Y. Zhang, S. Yuan, G. Day, X. Wang, X. Yang and H.-C. Zhou, Luminescent sensors based on metal-organic frameworks, Coord. Chem. Rev., 2018, 354, 28-45.

52 W. P. Lustig, S. Mukherjee, N. D. Rudd, A. V. Desai, J. Li and S. K. Ghosh, Metal-organic frameworks: functional luminescent and photonic materials for sensing applications, Chem. Soc. Rev., 2017, 46, 3242-3285.

53 B. Yan, Lanthanide-Functionalized Metal-Organic Framework Hybrid Systems To Create Multiple Luminescent Centers for Chemical Sensing, Acc. Chem. Res., 2017, 50, 2789-2798.

54 A. Karmakar, P. Samanta, A. V. Desai and S. K. Ghosh, Guest-Responsive Metal-Organic Frameworks as Scaffolds for Separation and Sensing Applications, Acc. Chem. Res., 2017, 50, 2457-2469.

55 Y. Liu, X.-Y. Xie, C. Cheng, Z.-S. Shao and H.-S. Wang, Strategies to fabricate metal-organic framework (MOF)- based luminescent sensing platforms, J. Mater. Chem. C, 2019, 7, 10743-10763.

56 A. Karmakar, P. Samanta, S. Dutta and S. K. Ghosh, Fluorescent "Turn-on" Sensing Based on Metal-Organic Frameworks (MOFs), Chem. - Asian J., 2019, 14, 4506-4519.

57 A. Carné, C. Carbonell, I. Imaza and D. Maspoch, Nanoscale metal-organic materials, Chem. Soc. Rev., 2011, 40, 291-305.

58 H. Xu, F. Liu, Y. Cui, B. Chen and G. Qian, A luminescent nanoscale metal-organic framework for sensing of nitroaromatic explosives, Chem. Commun., 2011, 47, 31533155.

59 Y. Lu, B. Yan and J.-L. Liu, Nanoscale metal-organic frameworks as highly sensitive luminescent sensors for $\mathrm{Fe}^{2+}$ in aqueous solution and living cells, Chem. Commun., 2014, 50, 9969-9972.

60 J. Aguilera-Sigalat and D. Bradshaw, Synthesis and applications of metal-organic framework-quantum dot (QD@MOF) composites, Coord. Chem. Rev., 2016, 307, 267-291.

61 R. Kaur, A. K. Paul and A. Deep, Nanocomposite of europium organic framework and quantum dots for highly sensitive chemosensing of trinitrotoluene, Forensic Sci. Int. , 2014, 242, 88-93.

62 X. Lin, G. Gao, L. Zheng, Y. Chi and G. Chen, Encapsulation of Strongly Fluorescent Carbon Quantum Dots in Metal-Organic Frameworks for Enhancing Chemical Sensing, Anal. Chem., 2014, 86, 1223-1228.

63 M. D. Allendorf, C. A. Bauer, R. K. Bhakta and R. J. T. Houk, Luminescent metal-organic frameworks, Chem. Soc. Rev., 2009, 38, 1330-1352.

64 R.-W. Huang, Y.-S. Wei, X.-Y. Dong, X.-H. Wu, C.-X. Du, S.-Q. Zang and T. C. W. Mak, Hypersensitive dual-function luminescence switching of a silver-chalcogenolate clusterbased metal-organic framework, Nat. Chem., 2017, DOI: 10.1038/nchem.2718.

65 United State Environment Protection Agency, 2014, 40 CFR Part 423, Appendix A, 653-654, https://www.govinfo. gov/content/pkg/CFR-2014-title40-vol29/pdf/CFR-2014-title 40-vol29-part423-appA.pdf.

66 P. Samanta, A. V. Desai, S. Sharma, P. Chandra and S. K. Ghosh, Selective Recognition of $\mathrm{Hg}^{2+}$ ion in Water by a Functionalized Metal-Organic Framework (MOF) Based Chemodosimeter, Inorg. Chem., 2018, 57, 2360-2364.

67 H. Tan, B. Liu and Y. Chen, Lanthanide Coordination Polymer Nanoparticles for Sensing of Mercury(II) by Photoinduced Electron Transfer, ACS Nano, 2012, 6, 10505-10511.

68 Y.-M. Zhu, C.-H. Zeng, T.-S. Chu, H.-M. Wang, Y.-Y. Yang, Y.-X. Tong, C.-Y. Su and W.-T. Wong, A novel highly luminescent LnMOF film: a convenient sensor for $\mathrm{Hg}^{2+}$ detecting, J. Mater. Chem. A, 2013, 1, 11312-11319.

69 L. Wen, X. Zheng, K. Lv, C. Wang and X. Xu, Two AminoDecorated Metal-Organic Frameworks for Highly Selective and Quantitatively Sensing of $\mathrm{Hg}^{\mathrm{II}}$ and $\mathrm{Cr}^{\mathrm{VI}}$ in Aqueous Solution, Inorg. Chem., 2015, 54, 7133-7135. 
70 P. Wu, Y. Liu, Y. Liu, J. Wang, Y. Li, W. Liu and J. Wang, Cadmium-Based Metal-Organic Framework as a Highly Selective and Sensitive Ratiometric Luminescent Sensor for Mercury(II), Inorg. Chem., 2015, 54, 1104611048.

71 N. D. Rudd, H. Wang, E. M. A. Fuentes-Fernandez, S. J. Teat, F. Chen, G. Hall, Y. J. Chabal and J. Li, Highly Efficient Luminescent Metal-Organic Framework for the Simultaneous Detection and Removal of Heavy Metals from Water, ACS Appl. Mater. Interfaces, 2016, 8, 3029430303.

72 J. He, M. Zha, J. Cui, M. Zeller, A. D. Hunter, S.-M. Yiu, S.-T. Lee and Z. Xu, Convenient Detection of $\mathrm{Pd}(\mathrm{II})$ by a Metal-Organic Framework with Sulfur and Olefin Functions, J. Am. Chem. Soc., 2013, 135, 7807-7810.

73 S. Sanda, S. Parshamoni, S. Biswas and S. Konar, Highly selective detection of palladium and picric acid by a luminescent MOF: a dual functional fluorescent sensor, Chem. Commun., 2015, 51, 6576-6579.

74 B. Parmar, Y. Rachuri, K. K. Bisht and E. Suresh, MixedLigand LMOF Fluorosensors for Detection of $\mathrm{Cr}(\mathrm{VI})$ Oxyanions and $\mathrm{Fe}^{3+} / \mathrm{Pd}^{2+}$ Cations in Aqueous Media, Inorg. Chem., 2017, 56, 10939-10949.

75 A. Helal, H. L. Nguyen, A. Al-Ahmed, K. E. Cordova and Z. H. Yamani, An Ultrasensitive and Selective MetalOrganic Framework Chemosensor for Palladium Detection in Water, Inorg. Chem., 2019, 58, 1738-1741.

76 R. A. Natour, Z. K. Ali, A. Assoud and M. Hmadeh, TwoDimensional Metal-Organic Framework Nanosheets as a Dual Ratiometric and Turn-off Luminescent Probe, Inorg. Chem., 2019, 58, 10912-10919.

77 Y. Wang, Y. Wua, J. Xie and X. Hu, Metal-organic framework modified carbon paste electrode for lead sensor, Sens. Actuators, B, 2013, 177, 1161-1166.

78 G. Ji, J. Liu, X. Gao, W. Sun, J. Z. Wang, S. Zhao and Z. Liu, A luminescent lanthanide MOF for selectively and ultra-high sensitively detecting $\mathrm{Pb}^{2+}$ ions in aqueous solution, J. Mater. Chem. A, 2017, 5, 10200-10205.

79 L. Cui, J. Wu, J. Li and H. Ju, Electrochemical Sensor for Lead Cation Sensitized with a DNA Functionalized Porphyrinic Metal-Organic Framework, Anal. Chem., 2015, 87, 10635-10641.

80 Y. Wang, H. Ge, Y. Wu, G. Ye, H. Chen and X. Hu, Construction of an electrochemical sensor based on amino-functionalized metal-organic frameworks for differential pulse anodic stripping voltammetric determination of lead, Talanta, 2014, 129, 100-105.

81 H. Guo, D. Wang, J. Chen, W. Weng, M. Huang and Z. Zheng, Simple fabrication of flake-like $\mathrm{NH}_{2}-\mathrm{MIL}-53(\mathrm{Cr})$ and its application as an electrochemical sensor for the detection of $\mathrm{Pb}^{2+}$, Chem. Eng. J., 2016, 289, 479-485.

82 Y. Wang, L. Wang, W. Huang, T. Zhang, X. Hu, J. A. Perman and S. Ma, A metal-organic framework and conducting polymer based electrochemical sensor for high performance cadmium ion detection, J. Mater. Chem. A, 2017, 5, 8385-8393.
83 M. Roushani, A. Valipour and Z. Saedi, Electroanalytical sensing of $\mathrm{Cd}^{2+}$ based on metal-organic framework modified carbon paste electrode, Sens. Actuators, B, 2016, 233, 419-425.

84 J.-N. Hao and B. Yan, A water-stable lanthanide-functionalized MOF as a highly selective and sensitive fluorescent probe for $\mathrm{Cd}^{2+}$, Chem. Commun., 2015, 51, 7737-7740.

85 X.-X. Jia, R.-X. Yao, F.-Q. Zhang and X.-M. Zhang, A Fluorescent Anionic MOF with $\mathrm{Zn}_{4}(\operatorname{trz})_{2}$ Chain for Highly Selective Visual Sensing of Contaminants: Cr(III) Ion and TNP, Inorg. Chem., 2017, 56, 2690-2696.

86 R. Lv, J. Wang, Y. Zhang, H. Li, L. Yang, S. Liao, W. Gu and X. Liu, An amino-decorated dual-functional metalorganic framework for highly selective sensing of $\mathrm{Cr}(\mathrm{iii})$ and $\mathrm{Cr}(\mathrm{vi})$ ions and detection of nitroaromatic explosives, J. Mater. Chem. A, 2016, 4, 15494-15500.

87 X.-Y. Guo, F. Zhao, J.-J. Liu, Z.-L. Liu and Y.-Q. Wang, An ultrastable zinc(ii)-organic framework as a recyclable multi-responsive luminescent sensor for $\mathrm{Cr}(\mathrm{iii}), \mathrm{Cr}(\mathrm{vi})$ and 4-nitrophenol in the aqueous phase with high selectivity and sensitivity, J. Mater. Chem. A, 2017, 5, 2003520043.

88 J. Liu, G. Ji, J. Xiao and Z. Liu, Ultrastable 1D Europium Complex for Simultaneous and Quantitative Sensing of $\mathrm{Cr}$ (III) and $\mathrm{Cr}$ (VI) Ions in Aqueous Solution with High Selectivity and Sensitivity, Inorg. Chem., 2017, 56, 41974205.

89 S.-R. Zhang, J. Li, D.-Y. Du, J.-S. Qin, S.-L. Li, W.-W. He, Z.-M. Su and Y.-Q. Lan, A multifunctional microporous anionic metal-organic framework for column-chromatographic dye separation and selective detection and adsorption of $\mathrm{Cr}^{3+}$, J. Mater. Chem. A, 2015, 3, 2342623434.

90 T.-Y. Gu, M. Dai, D. J. Young, Z.-G. Ren and J.-P. Lang, Luminescent Zn(II) Coordination Polymers for Highly Selective Sensing of $\mathrm{Cr}(\mathrm{III})$ and $\mathrm{Cr}(\mathrm{VI})$ in Water, Inorg. Chem., 2017, 56, 4668-4678.

91 J.-F. Feng, X. Yang, S.-Y. Gao, J. Shi and R. Cao, Facile and Rapid Growth of Nanostructured Ln-BTC Metal-Organic Framework Films by Electrophoretic Deposition for Explosives sensing in Gas and $\mathrm{Cr}^{3+}$ Detection in Solution, Langmuir, 2017, 33, 14238-14243.

92 Z. Zhan, X. Liang, X. Zhang, Y. Jia and M. Hu, A waterstable europium-MOF as a multifunctional luminescent sensor for some trivalent metal ions $\left(\mathrm{Fe}^{3+}, \mathrm{Cr}^{3+}, \mathrm{Al}^{3+}\right)$, $\mathrm{PO}_{4}{ }^{3-}$ ions, and nitroaromatic explosives, Dalton Trans., 2019, 48, 1786-1794.

93 X. Meng, M.-J. Wei, H.-N. Wang, H.-Y. Zang and Z.-Y. Zhou, Multifunctional luminescent Zn(II)-based metal-organic framework for high proton-conductivity and detection of $\mathrm{Cr}^{3+}$ ions in the presence of mixed metal ions, Dalton Trans., 2018, 47, 1383-1387.

94 Z. Sun, M. Yang, Y. Ma and L. Li, Multi-Responsive Luminescent Sensors Based on Two-Dimensional Lanthanide-Metal Organic Frameworks for Highly Selective and Sensitive Detection of $\mathrm{Cr}$ (III) and $\mathrm{Cr}(\mathrm{VI})$ Ions 
and Benzaldehyde, Cryst. Growth Des., 2017, 17, 43264335.

95 K. P. Carter, A. M. Young and A. E. Palmer, Fluorescent Sensors for Measuring Metal Ions in Living Systems, Chem. Rev., 2014, 114, 4564-4601.

96 X.-Y. Dong, R. Wang, J.-Z. Wang, S.-Q. Zang and T. C. W. Mak, Highly selective $\mathrm{Fe}^{3+}$ sensing and proton conduction in a water-stable sulfonate-carboxylate Tb-organic-framework, J. Mater. Chem. A, 2015, 3, 641647.

97 H. Xu, J. Gao, X. Qian, J. Wang, H. He, Y. Cui, Y. Yang, Z. Wang and G. Qian, Metal-organic framework nanosheets for fast-response and highly sensitive luminescent sensing of $\mathrm{Fe}^{3+}$, J. Mater. Chem. A, 2016, 4, 1090010905.

98 W. Yan, C. Zhang, S. Chen, L. Han and H. Zheng, Two Lanthanide Metal-Organic Frameworks as Remarkably Selective and Sensitive Bifunctional Luminescence Sensor for Metal Ions and Small Organic Molecules, ACS Appl. Mater. Interfaces, 2017, 9, 1629-1634.

99 P. C. Rao and S. Mandal, Europium-Based Metal-Organic Framework as a Dual Luminescence Sensor for the Selective Detection of the Phosphate Anion and $\mathrm{Fe}^{3+}$ Ion in Aqueous Media, Inorg. Chem., 2018, 57, 11855-11858.

100 B. Wang, Q. Yang, C. Guo, Y. Sun, L.-H. Xie and J.-R. Li, Stable $\operatorname{Zr}(\mathrm{IV})$-Based Metal-Organic Frameworks with Predesigned Functionalized Ligands for Highly Selective Detection of Fe(III) Ions in Water, ACS Appl. Mater. Interfaces, 2017, 9, 10286-10295.

101 A. Das and S. Biswas, A multi-responsive carbazole-functionalized $\mathrm{Zr}(\mathrm{IV})$-based metal-organic framework for selective sensing of Fe(III), cyanide and p-nitrophenol, Sens. Actuators, B, 2017, 250, 121-131.

102 R. Dalapati and S. Biswas, Aqueous Phase Sensing of $\mathrm{Fe}^{3+}$ and Ascorbic Acid by a Metal-Organic Framework and Its Implication in the Construction of Multiple Logic Gates, Chem. - Asian J., 2019, 14, 2822-2830.

103 J. Wang, Y. Fan, H.-W. Lee, C. Yi, C. Cheng, X. Zhao and M. Yang, Ultrasmall Metal-Organic Framework ZnMOF-74 Nanodots: Size Controlled Synthesis and Application for Highly Selective Colorimetric Sensing of Iron(III) in Aqueous Solution, ACS Appl. Nano Mater., 2018, 1, 3747-3753.

104 C.-S. Liu, M. Chen, J.-Y. Tian, L. Wang, M. Li, S.-M. Fang, X. Wang, L.-M. Zhou, Z.-W. Wang and M. Du, MetalOrganic Framework Supported on Processable Polymer Matrix by In Situ Copolymerization for Enhanced Iron(III) Detection, Chem. - Eur. J., 2017, 23, 3885-3890.

105 S. Let, P. Samanta, S. Dutta and S. K. Ghosh, A Dye@MOF composite as luminescent sensory material for selective and sensitive recognition of $\mathrm{Fe}(\mathrm{III})$ ions in water, Inorg. Chim. Acta, 2020, 500, 119205.

106 S. Fajal, P. Samanta, S. Dutta and S. K. Ghosh, Selective and sensitive recognition of $\mathrm{Fe}^{3+}$ ion by a Lewis basic functionalized chemically stable metal-organic framework (MOF), Inorg. Chim. Acta, 2020, 502, 119359.
107 L. Liu, Y. Wang, R. Lin, Z. Yao, Q. Lin, L. Wang, Z. Zhang and S. Xiang, Two water-stable lanthanide metal-organic frameworks with oxygen-rich channels for fluorescence sensing of Fe(III) ions in aqueous solution, Dalton Trans., 2018, 47, 16190-16196.

108 C.-H. Chen, X.-S. Chen, L. Li, Y.-B. Huang and R. Cao, Highly selective sensing of $\mathrm{Fe}^{3+}$ by an anionic metalorganic framework containing uncoordinated nitrogen and carboxylate oxygen sites, Dalton Trans., 2018, 47, 3452-3458.

109 H. Chen, P. Fan, X. Tu, H. Min, X. Yu, X. Li, J.-L. Zeng, S. Zhang and P. Cheng, A Bifunctional Luminescent Metal-Organic Framework for the Sensing of Paraquat and $\mathrm{Fe}^{3+}$ Ions in Water, Chem. - Asian J., 2019, 14, 36113619.

110 W.-H. Huang, J. Ren, Y.-H. Yang, X.-M. Li, Q. Wang, N. Jiang, J.-Q. Yu, F. Wang and J. Zhang, Water-Stable Metal-Organic Frameworks with Selective Sensing on $\mathrm{Fe}^{3+}$ and Nitroaromatic Explosives, and Stimuli-Responsive Luminescence on Lanthanide Encapsulation, Inorg. Chem., 2019, 58, 1481-1491.

111 Y. Lu, B. Yan and J.-L. Liu, Nanoscale metal-organic frameworks as highly sensitive luminescent sensors for $\mathrm{Fe}^{2+}$ in aqueous solution and living cells, Chem. Commun., 2014, 50, 9969-9972.

112 R. Krämer, Fluorescent Chemosensors for $\mathrm{Cu}^{2+}$ Ions: Fast, Selective, and Highly Sensitive, Angew. Chem., Int. Ed., 1998, 37, 772-773.

113 B. P. Zietz, J. D. de Vergara and H. Dunkelberg, Copper concentrations in tap water and possible effects on infant's health-Results of a study in Lower Saxony, Germany, Environ. Res., 2003, 92, 129-138.

114 X. Lin, G. Gao, L. Zheng, Y. Chi and G. Chen, Encapsulation of Strongly Fluorescent Carbon Quantum Dots in Metal-Organic Frameworks for Enhancing Chemical Sensing, Anal. Chem., 2014, 86, 1223-1228.

115 S. Bhattacharyya, A. Chakraborty, K. Jayaramulu, A. Hazra and T. K. Maji, A bimodal anionic MOF: turn-off sensing of $\mathrm{Cu}^{\mathrm{II}}$ and specific sensitization of $\mathrm{Eu}^{\mathrm{III}}$, Chem. Commun., 2014, 50, 13567-13570.

116 Y.-Z. Chen and H.-L. Jiang, Porphyrinic Metal-Organic Framework Catalyzed Heck-Reaction: Fluorescence "TurnOn” Sensing of Cu(II) Ion, Chem. Mater., 2016, 28, 66986704.

117 L. Li, S. Shen, R. Lin, Y. Bai and H. Liu, Rapid and specific luminescence sensing of $\mathrm{Cu}(\mathrm{II})$ ions with a porphyrinic metal-organic framework, Chem. Commun., 2017, 53, 9986-9989.

118 C. Qiao, X. Qu, Q. Yang, Q. Wei, G. Xie, S. Chen and D. Yang, Instant high-selectivity Cd-MOF chemosensor for naked-eye detection of $\mathrm{Cu}(\mathrm{II})$ confirmed using in situ microcalorimetry, Green Chem., 2016, 18, 951-956.

119 J. Hao, F. Liu, N. Liu, M. Zeng, Y. Song and L. Wang, Ratiometric fluorescent detection of $\mathrm{Cu}^{2+}$ with carbon dots chelated Eu-based metal-organic frameworks, Sens. Actuators, B, 2017, 245, 641-647. 
120 Y.-W. Li, J.-R. Li, L.-F. Wang, B.-Y. Zhou, Q. Chen and X.-H. Bu, Microporous metal-organic frameworks with open metal sites as sorbents for selective gas adsorption and fluorescence sensors for metal ions, J. Mater. Chem. A, 2013, 1, 495-499.

121 Z. Hao, X. Song, M. Zhu, X. Meng, S. Zhao, S. Su, W. Yang, S. Song and H. Zhang, One-dimensional channel-structured Eu-MOF for sensing small organic molecules and $\mathrm{Cu}^{2+}$ ion, J. Mater. Chem. A, 2013, 1, 11043-11050.

122 B. Liu, W.-P. Wu, L. Hou and Y.-Y. Wang, Four uncommon nanocage-based Ln-MOFs: highly selective luminescent sensing for $\mathrm{Cu}^{2+}$ ions and selective $\mathrm{CO}_{2}$ capture, Chem. Commun., 2014, 50, 8731-8734.

123 J. Ye, L. Zhao, R. F. Bogale, Y. Gao, X. Wang, X. Qian, S. Guo, J. Zhao and G. Ning, Highly Selective Detection of 2,4,6-Trinitrophenol and $\mathrm{Cu}^{2+}$ Ions Based on a Fluorescent Cadmium-Pamoate Metal-Organic Framework, Chem. - Eur. J., 2015, 21, 2029-2037.

124 Z. Chen, Y. Sun, L. Zhang, D. Sun, F. Liu, Q. Meng, R. Wang and D. Sun, A tubular europium-organic framework exhibiting selective sensing of $\mathrm{Fe}^{3+}$ and $\mathrm{Al}^{3+}$ over mixed metal ions, Chem. Commun., 2013, 49, 1155711559.

125 L.-H. Cao, F. Shi, W.-M. Zhang, S.-Q. Zang and T. C. W. Mak, Selective Sensing of $\mathrm{Fe}^{3+}$ and $\mathrm{Al}^{3+}$ Ions and Detection of 2,4,6-Trinitrophenol by a Water-Stable Terbium-Based Metal-Organic Framework, Chem. - Eur. J., 2015, 21, 15705-15712.

126 M.-H. Yu, T.-L. Hu and X.-H. Bu, A metal-organic framework as a "turn on" fluorescent sensor for aluminum ions, Inorg. Chem. Front., 2017, 4, 256-260.
127 F. Rouhani, F. Rafizadeh-Masuleha and A. Morsali, Selective sacrificial metal-organic frameworks: a highly quantitative colorimetric naked-eye detector for aluminum ions in aqueous solutions, J. Mater. Chem. A, 2019, 7, 18634-18641.

128 W.-M. Chen, X.-L. Meng, G.-L. Zhuang, Z. Wang, M. Kurmoo, Q.-Q. Zhao, X.-P. Wang, B. Shan, C.-H. Tung and D. Sun, A superior fluorescent sensor for $\mathrm{Al}^{3+}$ and $\mathrm{UO}_{2}{ }^{2+}$ based on a $\mathrm{Co}(\mathrm{II})$ metal-organic framework with exposed pyrimidyl Lewis base sites, J. Mater. Chem. A, 2017, 5, 13079-13085.

129 J.-N. Hao and B. Yan, $\mathrm{Ag}^{+}$-sensitized lanthanide luminescence in $\mathrm{Ln}^{3+}$ post-functionalized metal-organic frameworks and $\mathrm{Ag}^{+}$sensing, J. Mater. Chem. A, 2015, 3, 47884792 .

130 F. Kamel, Paths from Pesticides to Parkinson's, Science, 2013, 341, 722-723.

131 W. Du, Z. Zhu, Y.-L. Bai, Z. Yang, S. Zhu, J. Xu, Z. Xie and J. Fanga, An anionic sod-type terbium-MOF with extralarge cavities for effective anthocyanin extraction and methyl viologen detection, Chem. Commun., 2018, 54, 5972-5975.

132 M. E. Mahmoud, Z. Moussa, T. Prakasam, L. Li, M. G. Abiad, D. Patra and M. Hmadeh, Lanthanides based metal organic frameworks for luminescence sensing of toxic metal ions, J. Solid State Chem., 2020, 281, 121031.

133 Y.-Y. Jia, Y.-H. Zhang, J. Xu, R. Feng, M.-S. Zhang and X.-H. Bu, A high-performance "sweeper" for toxic cationic herbicides: an anionic metal-organic framework with a tetrapodal cage, Chem. Commun., 2015, 51, 1743917442. 